\title{
Enantioselective Synthesis of a PKC Inhibitor via Catalytic C-H Bond Activation
}

Rebecca M. Wilson, Reema K. Thalji, Robert G. Bergman, ${ }^{*}$ and Jonathan A. Ellman*

Department of Chemistry and Division of Chemical Sciences, Lawrence Berkeley National Laboratory, University of California, Berkeley, CA 94720

\section{Supporting Information}

I. Spectroscopic and Analytical Data

Page \#

II. Structural Data for $\mathbf{1 2}$

III. $\quad{ }^{1} \mathrm{H},{ }^{13} \mathrm{C}$, and ${ }^{19} \mathrm{~F}$ NMR Spectra 


\section{Spectroscopic and Analytical Data}

General Experimental. Unless otherwise noted, all materials were obtained from commercial sources and used without further purification. Benzene, toluene, THF, diethyl ether, pentane, and hexanes were passed through columns of activated alumina under nitrogen prior to use. Anhydrous DMF was cannula-transferred to a glass bomb under nitrogen and stored over molecular sieves. Methanol was distilled from magnesium methoxide immediately prior to use. All organic reactions were performed in flame- or oven-dried glassware under nitrogen unless otherwise noted. Organic solutions were concentrated on a rotary evaporator. The following compounds were prepared according to literature procedures: $\left[\mathrm{RhCl}(\operatorname{coe})_{2}\right]_{2}{ }^{1}$ and ligands $\mathbf{L}$ and L'. ${ }^{2}$ All C-H activation reactions were assembled in a Braun inert atmosphere glove box, and cyclization enantioselectivities were determined by chiral HPLC analysis performed on a Shimadzu 10A-VP series instrument. Melting points were measured on a Thomas Hoover UniMelt capillary melting point apparatus and are uncorrected. IR spectra were recorded on a Thermo Nicolet Avatar 370 fitted with a single bounce ZnSe ATR plate; stretching frequencies are reported in $\mathrm{cm}^{-1}$, and the data shown include only major absorbtions. ${ }^{1} \mathrm{H},{ }^{13} \mathrm{C}$, and ${ }^{19} \mathrm{~F}$ NMR spectra were recorded on Bruker AV-300, AVQ-400, or DRX-500 instruments, as noted. NMR chemical shifts are reported in ppm and referenced to the residual protio solvent signal; coupling constants are reported in $\mathrm{Hz}$. Elemental analyses and mass spectra were obtained by the University of California, Berkeley Microanalytical Services facility.

1-Allyl-5-methoxy-1H-indole-3-carboxaldehyde (3). To a round bottom flask fitted with a condenser and a stir bar was added 5-methoxyindole-3-carboxaldehyde (1.00 g, 5.71 mmol), potassium carbonate $(3.85 \mathrm{~g}, 28.5 \mathrm{mmol})$, acetone $(57 \mathrm{~mL}, 0.1 \mathrm{M})$, and allyl bromide $(2.47 \mathrm{~mL}, 28.5 \mathrm{mmol})$. The reaction mixture was heated to reflux for $6 \mathrm{~h}$, after which it was allowed to cool to room temperature, filtered, and concentrated. Chromatography on silica gel $\left(5 \% \mathrm{EtOAc} / \mathrm{CH}_{2} \mathrm{Cl}_{2}\right)$ afforded the title compound as a white crystalline solid (1.19 g, 97\%). Mp: 81-82 ${ }^{\circ} \mathrm{C}$. IR: $1647 \mathrm{~cm}^{-1} .{ }^{1} \mathrm{H}$ NMR $\left(400 \mathrm{MHz}, \mathrm{C}_{6} \mathrm{D}_{6}\right): \delta 10.11(\mathrm{~s}, 1 \mathrm{H}), 8.36(\mathrm{~d}, J=2.3 \mathrm{~Hz}, 1 \mathrm{H})$, $7.15(\mathrm{dd}, J=2.6,9.3 \mathrm{~Hz}, 1 \mathrm{H}), 6.85$ (d, $J=8.8,1 \mathrm{H}), 6.69$ (s, 1H), 5.37 (ddd, $J=5.6,11.0,22.3$ $\mathrm{Hz}, 1 \mathrm{H}), 4.87$ (dd, $J=1.1,10.5 \mathrm{~Hz}, 1 \mathrm{H}), 4.69$ (dd, $J=1.1,17.2 \mathrm{~Hz}, 1 \mathrm{H}), 3.80$ (d, $J=5.4 \mathrm{~Hz}$, 2H), $3.54(\mathrm{~s}, 3 \mathrm{H}) .{ }^{13} \mathrm{C}\left\{{ }^{1} \mathrm{H}\right\} \mathrm{NMR}\left(100 \mathrm{MHz}, \mathrm{CDCl}_{3}\right): \delta 184.5,156.6,138.4,132.1,131.7,126.1$, 119.0, 118.1, 114.5, 111.1, 103.3, 55.8, 49.7. Anal. Calcd. for $\mathrm{C}_{13} \mathrm{H}_{13} \mathrm{NO}_{2}: \mathrm{C}, 72.54 ; \mathrm{H}, 6.09 ; \mathrm{N}$, 6.51. Found: C, 72.31; H, 5.90; N, 6.41. 
Benzyl-(1-allyl-5-methoxy-1H-indol-3-ylmethylene)-amine. (13) In an inert atmosphere glove box, benzylamine $(0.560 \mathrm{~mL}, 5.11 \mathrm{mmol})$ was added to a solution of $\mathbf{3}(1.00 \mathrm{~g}$, $4.65 \mathrm{mmol})$ in benzene $(9 \mathrm{~mL}, 0.5 \mathrm{M})$. The reaction mixture was stirred for $12 \mathrm{~h}$ over $3 \mathrm{~A}$ molecular sieves $(5 \mathrm{~g})$ at room temperature, after which it was filtered through celite and concentrated. The product was obtained as a pale yellow oil in quantitative yield $(1.41 \mathrm{~g})$, which was stored in the glove box freezer $\left(-35^{\circ} \mathrm{C}\right)$ and used without further purification in the next step. ${ }^{1} \mathrm{H}$ NMR (400 MHz, toluene- $\left.d_{8}\right): \delta 8.37(\mathrm{~d}, J=2.5 \mathrm{~Hz}, 1 \mathrm{H}), 8.33(\mathrm{~s}, 1 \mathrm{H}), 7.44(\mathrm{~d}, J=7.3$ $\mathrm{Hz}, 2 \mathrm{H}), 7.22$ (t, $J=7.5 \mathrm{~Hz}, 2 \mathrm{H}), 7.10$ (d, $J=7.5 \mathrm{~Hz}, 1 \mathrm{H}), 7.01$ (dd, $J=2.6,8.9 \mathrm{~Hz}, 1 \mathrm{H}), 6.87$ $(\mathrm{d}, J=8.8 \mathrm{~Hz}, 1 \mathrm{H}), 6.73(\mathrm{~s}, 1 \mathrm{H}), 5.48(\mathrm{ddd}, J=5.3,10.6,22.1 \mathrm{~Hz}, 1 \mathrm{H}), 4.85$ (dd, $J=1.2,10.4$ $\mathrm{Hz}, 1 \mathrm{H}), 4.77$ (s, 2H), 4.72 (dd, $J=1.3,17.1 \mathrm{~Hz}, 1 \mathrm{H}), 3.95$ (d, $J=5.5 \mathrm{~Hz}, 2 \mathrm{H}), 3.57$ (s, 3H). ${ }^{13} \mathrm{C}\left\{{ }^{1} \mathrm{H}\right\}$ NMR $\left(100 \mathrm{MHz}, \mathrm{CDCl}_{3}\right): \delta 156.1,155.5,140.9,132.8,132.3,132.2,128.4,127.8$, $126.9,126.6,118.0,114.4,113.3,110.6,103.8,65.5,55.9,49.3$.

6-Methoxy-2,3-dihydro-1H-3a-aza-cyclopenta[a]indene-8-carboxaldehyde (4). To a glass vessel equipped with a vacuum stopcock and a stir bar was added 13 (500 mg, $1.64 \mathrm{mmol}$ ), $\mathrm{RhCl}\left(\mathrm{PPh}_{3}\right)_{3}(76.0 \mathrm{mg}, 0.0821 \mathrm{mmol})$, and toluene $(16 \mathrm{~mL}, 0.1 \mathrm{M})$. The solution was heated at $125^{\circ} \mathrm{C}$ for $1.5 \mathrm{~h}$, after which time it was concentrated and chromatographed on silica gel (30\% EtOAc/hexanes) to afford the title compound as a white solid in 81\% yield (287 mg). Mp: 150$151{ }^{\circ} \mathrm{C}$. IR: $1632 \mathrm{~cm}^{-1} .{ }^{1} \mathrm{H}$ NMR $\left(400 \mathrm{MHz}, \mathrm{CDCl}_{3}\right): \delta 9.86(\mathrm{~s}, 1 \mathrm{H}), 7.70(\mathrm{~d}, J=1.7 \mathrm{~Hz}, 1 \mathrm{H})$, $7.08(\mathrm{~d}, J=8.7 \mathrm{~Hz}, 1 \mathrm{H}), 6.83(\mathrm{dd}, J=2.3,8.9,1 \mathrm{H}), 4.00(\mathrm{t}, J=7.1 \mathrm{~Hz}, 2 \mathrm{H}), 3.87(\mathrm{~s}, 3 \mathrm{H}), 3.16(\mathrm{t}$, $J=7.6 \mathrm{~Hz}, 2 \mathrm{H}), 2.64$ (quint, $J=7.3 \mathrm{~Hz}, 2 \mathrm{H}) .{ }^{13} \mathrm{C}\left\{{ }^{1} \mathrm{H}\right\} \mathrm{NMR}(100 \mathrm{MHz}): \delta 183.2,156.5,155.9$, 130.5, 128.0, 112.5, 110.7, 110.1, 103.5, 55.8, 44.6, 26.6, 24.4. Anal. Calcd. for $\mathrm{C}_{13} \mathrm{H}_{13} \mathrm{NO}_{2}$ : C, 72.54; H, 6.09; N, 6.51. Found: C, 72.27; H, 6.00; N, 6.39.

6-Methoxy-8-(2-nitro-propenyl)-2,3-dihydro-1H-3a-aza-cyclopenta[a]indene $\quad(14){ }^{3}$

To a round bottom flask fitted with a condenser and a stir bar was added 4 (198 mg, 0.920 $\mathrm{mmol})$, ammonium acetate $(80.2 \mathrm{mg}, 1.04 \mathrm{mmol})$ and nitroethane $(2 \mathrm{~mL}, 0.5 \mathrm{M})$. The reaction mixture was heated at $100{ }^{\circ} \mathrm{C}$ for $1 \mathrm{~h}$, after which time it was cooled to room temperature and diluted with $6 \mathrm{~mL}$ of water. The resulting mixture was extracted with EtOAc $(3 \times 5 \mathrm{~mL})$, and the combined organic extracts were washed with brine, dried over sodium sulfate, and concentrated. Chromatography on silica gel $(10 \%$ EtOAc/heptane) afforded the title compound as a bright orange solid in 95\% yield (237 mg). ${ }^{1} \mathrm{H}$ NMR (400 $\left.\mathrm{MHz}, \mathrm{CDCl}_{3}\right): \delta 8.40(\mathrm{~s}, 1 \mathrm{H}), 7.17(\mathrm{~d}, J=$ $8.8 \mathrm{~Hz}, 1 \mathrm{H}), 7.06(\mathrm{~d}, J=2.2 \mathrm{~Hz}, 1 \mathrm{H}), 6.88(\mathrm{dd}, J=2.2,8.9 \mathrm{~Hz}, 1 \mathrm{H}), 4.14(\mathrm{t}, J=7.1 \mathrm{~Hz}, 2 \mathrm{H})$, 
$3.88(\mathrm{~s}, 3 \mathrm{H}), 3.11(\mathrm{t}, J=7.2 \mathrm{~Hz}, 2 \mathrm{H}), 2.69$ (quint, $J=7.1 \mathrm{~Hz}, 2 \mathrm{H}), 2.43(\mathrm{~s}, 3 \mathrm{H}),{ }^{13} \mathrm{C}\left\{{ }^{1} \mathrm{H}\right\} \mathrm{NMR}$ (100 MHz): $\delta 155.3,153.8,148.2,140.6,132.4,128.9,111.9,110.9,101.8,101.6,55.8,44.7$, 27.3, 26.5, 15.6. Anal. Calcd. for $\mathrm{C}_{15} \mathrm{H}_{16} \mathrm{~N}_{2} \mathrm{O}_{3}$ : C, 66.16; H, 5.92; N, 10.29. Found: C, 66.07; H, $5.96 ; \mathrm{N}, 10.13$.

\section{2-(6-Methoxy-2,3-dihydro-1H-3a-aza-cyclopenta $[a]$ inden-8-yl)-1-methyl-ethylamine}

fumarate (1). ${ }^{4}$ To a stirred solution of lithium aluminum hydride $(41.8 \mathrm{mg}, 1.10 \mathrm{mmol})$ in THF (3.1 mL, 0.35 M) was added dropwise a solution of $14(200 \mathrm{mg}, 0.735 \mathrm{M})$ in THF (4 mL, 0.2 M). The reaction mixture was heated to reflux for $4 \mathrm{~h}$, after which time it was cooled to $0{ }^{\circ} \mathrm{C}$, and the reaction was quenched by dropwise addition of saturated aqueous sodium potassium tartrate solution $(20 \mathrm{~mL})$. The mixture was stirred for $30 \mathrm{~min}$ and filtered through celite. The filtrate was extracted with $\mathrm{CH}_{2} \mathrm{Cl}_{2}(3 \times 15 \mathrm{~mL})$, and the combined organic extracts were washed with water and brine, dried over magnesium sulfate, and concentrated. The residue was dissolved in 2-propanol (0.4 mL) and added to a solution of fumaric acid (694 mg, $0.613 \mathrm{mmol}$ ) in 2propanol $(8 \mathrm{~mL})$ at $50{ }^{\circ} \mathrm{C}$. The solution was cooled to $0{ }^{\circ} \mathrm{C}$ and allowed to stand at that temperature for $12 \mathrm{~h}$. The solution was then filtered, and the resulting solid was washed with 2propanol and diethyl ether and dried to afford the title compound as a white solid in 59\% yield (106 mg). ${ }^{1} \mathrm{H}$ NMR (400 MHz, DMSO-d $): \delta 7.17(\mathrm{~d}, J=8.6 \mathrm{~Hz}, 1 \mathrm{H}), 7.03(\mathrm{~d}, J=7.03 \mathrm{~Hz}, 1 \mathrm{H})$, $6.68(\mathrm{dd}, J=2.5,8.8 \mathrm{~Hz}, 1 \mathrm{H}), 6.51$ (s, 2H), 4.00 (t, $J=7.1 \mathrm{~Hz}, 2 \mathrm{H}), 3.76(\mathrm{~s}, 3 \mathrm{H}), 3.39$ (m, 2H), $3.00-2.86(\mathrm{~m}, 3 \mathrm{H}), 2.73(\mathrm{dd}, J=8.3,14.2,1 \mathrm{H}), 2.53(\mathrm{dd}, J=7.3,14.2 \mathrm{~Hz}, 1 \mathrm{H}), 1.16(\mathrm{~d}, J=6.6$ $\mathrm{Hz}, 3 \mathrm{H}) .{ }^{13} \mathrm{C}\left\{{ }^{1} \mathrm{H}\right\}$ NMR $(100 \mathrm{MHz}): \delta 167.9,153.7,144.4,135.4,133.0,128.1,110.7,109.8$, 101.2, 99.8, 55.9, 48.0, 44.2, 30.4, 27.7, 23.6, 18.7. EI-HRMS Calcd. for $\mathrm{C}_{15} \mathrm{H}_{20} \mathrm{~N}_{2} \mathrm{O}[\mathrm{M}]^{+}$: 244.1576. Found: 244.1570.

3-Chloro-2-methoxymethylpropene (5). A solution of sodium hydride $(60 \%$ dispersion in mineral oil, $506 \mathrm{mg}, 12.7 \mathrm{mmol})$ in methanol $(12.7 \mathrm{~mL}, 0.1 \mathrm{M})$ was stirred for 20 min at room temperature, after which time 3-chloro-2-chloromethylpropene (2.00 mL, $19.0 \mathrm{mmol}, 1.5 \mathrm{equiv})$ was added. The flask was sealed and allowed to stir for $18 \mathrm{~h}$. The resulting suspension was then filtered, diluted with $20 \mathrm{~mL}$ of water, and extracted with pentane $(2 \times 40 \mathrm{~mL})$. The combined pentane extracts were dried over magnesium sulfate, concentrated to a volume of approximately $6 \mathrm{~mL}$, and chromatographed on a short silica gel column (starting material eluted with pentane, product eluted with $1 \%$ diethyl ether/pentane). Unreacted 3-chloro-2-chloromethylpropene was recovered $(1.01 \mathrm{~g})$, and the desired product was obtained as a colorless oil $(0.495 \mathrm{~g}, 22 \%$ based 
on 3-chloro-2-chloromethylpropene, $40 \%$ based on recovered starting material). ${ }^{1} \mathrm{H}$ NMR (300 $\left.\mathrm{MHz}, \mathrm{C}_{6} \mathrm{D}_{6}\right): \delta 5.06(\mathrm{~s}, 1 \mathrm{H}), 5.00(\mathrm{~s}, 1 \mathrm{H}), 3.82(\mathrm{~s}, 2 \mathrm{H}), 3.80(\mathrm{~s}, 2 \mathrm{H}), 3.07(\mathrm{~s}, 3 \mathrm{H}) .{ }^{13} \mathrm{C}\left\{{ }^{1} \mathrm{H}\right\} \mathrm{NMR}$ $(100 \mathrm{MHz}): \delta 142.7,115.9,72.5,57.9,45.2$. EI-HRMS Calcd. for $\mathrm{C}_{5} \mathrm{H}_{9} \mathrm{Cl}[\mathrm{M}-\mathrm{Cl}]^{+}: 85.0653$. Found: 85.0655.

1-(2-Methoxymethyl-allyl)-1 $\boldsymbol{H}$-indole-3-carboxaldehyde (6). To a 3-neck round bottom flask fitted with a condenser and a stir bar was added indole-3-carboxaldehyde $(0.960 \mathrm{~g}$, $6.64 \mathrm{mmol})$, potassium carbonate $(1.80 \mathrm{~g}, 13.3 \mathrm{mmol})$, anhydrous DMF (30 mL, $0.2 \mathrm{M})$, and 5 $(0.880 \mathrm{~g}, 7.30 \mathrm{mmol})$. The resulting mixture was heated to $50{ }^{\circ} \mathrm{C}$ overnight $(12 \mathrm{~h})$, after which time it was cooled to room temperature, diluted with $100 \mathrm{~mL}$ of $2: 1$ hexanes: $\mathrm{CH}_{2} \mathrm{Cl}_{2}$, and washed with water $(3 \times 100 \mathrm{~mL})$ to remove the DMF. The organic layer was washed with brine, dried over magnesium sulfate, concentrated, and chromatographed on silica gel (50\% EtOAc/hexanes) to give a pale pink solid, which was subsequently recrystallized from pentane/toluene by vapor diffusion at $-35{ }^{\circ} \mathrm{C}$ to give a colorless crystalline solid in $64 \%$ yield $(0.970 \mathrm{~g})$. Mp: $51.5-52.5{ }^{\circ} \mathrm{C}$. IR: $1645 \mathrm{~cm}^{-1} .{ }^{1} \mathrm{H}$ NMR $\left(300 \mathrm{MHz}, \mathrm{CDCl}_{3}\right): \delta 9.98(\mathrm{~s}, 1 \mathrm{H}), 8.34-8.26(\mathrm{~m}, 1 \mathrm{H}), 7.71(\mathrm{~s}, 1 \mathrm{H})$, 7.38-7.26 (m, 3H), $5.22(\mathrm{~s}, 1 \mathrm{H}), 4.99(\mathrm{~s}, 1 \mathrm{H}), 4.80(\mathrm{~s}, 2 \mathrm{H}), 3.77(\mathrm{~s}, 2 \mathrm{H}), 3.30(\mathrm{~s}, 3 \mathrm{H}) .{ }^{13} \mathrm{C}\left\{{ }^{1} \mathrm{H}\right\}$ NMR (75 MHz): $\delta 184.9,140.9,139.4,137.7,125.6,124.4,123.2,122.4,118.7,116.4,110.7$, 73.5, 58.4, 49.6. Anal. Calcd. for $\mathrm{C}_{14} \mathrm{H}_{15} \mathrm{NO}_{2}$ : $\mathrm{C}, 73.34 ; \mathrm{H}, 6.59 ; \mathrm{N}, 6.11$. Found: $\mathrm{C}, 73.38 ; \mathrm{H}$, $6.64 ; \mathrm{N}, 6.03$.

General Procedure for Imine Synthesis. In an inert atmosphere glove box, the appropriate amine (1.1 equiv) was added to a solution of 6 in benzene $(0.5 \mathrm{M})$. The reaction mixture was stirred over $3 \mathrm{~A}$ molecular sieves $(1.00 \mathrm{~g}$ sieves $/ 1.00 \mathrm{mmol}$ 6) for $12 \mathrm{~h}$ at room temperature, after which time it was diluted with an equal volume of diethyl ether, filtered through Celite ${ }^{\circledR}$, and concentrated in vacuo. The purity of the imine was verified by ${ }^{1} \mathrm{H}$ NMR and, with the exception of $\mathbf{8}$, used immediately without further purification.

Benzyl-[1-(2-methoxymethyl-allyl)-1H-indol-3-ylmethylene]-amine. This compound was prepared according to the general procedure for imine synthesis from $6(0.600 \mathrm{~g}, 2.62 \mathrm{mmol})$ and benzylamine $(0.310 \mathrm{~g}, 2.88 \mathrm{mmol})$. The product was isolated as a white solid in $94 \%$ yield $(0.782 \mathrm{~g}) .{ }^{1} \mathrm{H}$ NMR $\left(400 \mathrm{MHz}\right.$, toluene- $\left.d_{8}\right): \delta 8.89(\mathrm{~d}, J=7.3 \mathrm{~Hz}, 1 \mathrm{H}), 8.40(\mathrm{~s}, 1 \mathrm{H}), 7.48$ (d, $J=$ 7.6 Hz, 2H), 7.28-7.10 (m, 6H), $6.91(\mathrm{~s}, 1 \mathrm{H}), 4.87$ (s, 1H), $4.80(\mathrm{~s}, 2 \mathrm{H}), 4.62(\mathrm{~s}, 1 \mathrm{H}), 4.27$ (s, 2H), 3.39 (s, 2H), 2.98 (s, 3H). 
4-Methoxybenzyl-[1-(2-methoxymethyl-allyl)-1 $H$-indol-3-ylmethylene]-amine. This compound was prepared according to the general procedure for imine synthesis from 6 (100 mg, $0.436 \mathrm{mmol})$ and 4-methoxybenzylamine $(65.8 \mathrm{mg}, 0.480 \mathrm{mmol})$ in $94 \%$ yield $(143 \mathrm{mg}) .{ }^{1} \mathrm{H}$ NMR (400 MHz, toluene- $\left.d_{8}\right): \delta 8.87(\mathrm{~d}, J=7.7 \mathrm{~Hz}, 1 \mathrm{H}), 8.38(\mathrm{~s}, 1 \mathrm{H}), 7.35(\mathrm{~d}, J=8.2,2 \mathrm{H}), 7.21$ (m, 2H), $7.11(\mathrm{~d}, J=8.3,1 \mathrm{H}), 6.88(\mathrm{~s}, 1 \mathrm{H}), 6.80(\mathrm{~d}, J=8.1 \mathrm{~Hz}, 2 \mathrm{H}), 4.84(\mathrm{~s}, 1 \mathrm{H}), 4.76(\mathrm{~s}, 2 \mathrm{H})$, $4.59(\mathrm{~s}, 1 \mathrm{H}), 4.24(\mathrm{~s}, 2 \mathrm{H}), 3.36(\mathrm{~s}, 5 \mathrm{H}), 2.95(\mathrm{~s}, 3 \mathrm{H})$.

\section{4-(Trifluoromethyl)benzyl-[1-(2-methoxymethyl-allyl)-1 $H$-indol-3-ylmethylene]-}

amine. This compound was prepared according to the general procedure for imine synthesis from 6 (100 mg, $0.436 \mathrm{mmol})$ and 4-(trifluoromethyl)benzylamine $(84.0 \mathrm{mg}, 0.480 \mathrm{mmol})$ in 95\% yield (160 mg). ${ }^{1} \mathrm{H}$ NMR (400 MHz, toluene- $\left.d_{8}\right): \delta 8.84(\mathrm{~d}, J=7.8 \mathrm{~Hz}, 1 \mathrm{H}), 8.28(\mathrm{~s}, 1 \mathrm{H})$, $7.36(\mathrm{~d}, J=7.8 \mathrm{~Hz}, 2 \mathrm{H}), 7.27(\mathrm{~m}, 3 \mathrm{H}), 7.21(\mathrm{t}, J=7.8 \mathrm{~Hz}, 1 \mathrm{H}), 7.14,(\mathrm{~d}, J=7.6 \mathrm{~Hz}, 1 \mathrm{H}), 6.91(\mathrm{~s}$, $1 \mathrm{H}), 4.84(\mathrm{~s}, 1 \mathrm{H}), 4.60(\mathrm{~s}, 3 \mathrm{H}), 4.25(\mathrm{~s}, 2 \mathrm{H}) 3.36(\mathrm{~s}, 2 \mathrm{H}), 2.96(\mathrm{~s}, 3 \mathrm{H})$.

\section{3,5-Bis(trifluoromethyl)benzyl-[1-(2-methoxymethyl-allyl)-1 $H$-indol-3-ylmethylene]-}

amine (8). This compound was prepared according to the general procedure for imine synthesis from 6 (1.02 g, $4.45 \mathrm{mmol})$ and 3,5-bis(trifluoromethyl)benzylamine $1.19 \mathrm{~g}, 4.89 \mathrm{mmol})$. The crude product was recrystallized from pentane at $-35^{\circ} \mathrm{C}$ to provide the title compound as a white solid in $85 \%$ yield $(1.78 \mathrm{~g}) .{ }^{1} \mathrm{H}$ NMR (400 MHz, toluene- $\left.d_{8}\right): \delta 8.72(\mathrm{~d}, J=7.6 \mathrm{~Hz}, 1 \mathrm{H}), 8.23(\mathrm{~s}$, 1H), $7.79(\mathrm{~s}, 2 \mathrm{H}), 7.71(\mathrm{~s}, 1 \mathrm{H}), 7.24-7.15(\mathrm{~m}, 2 \mathrm{H}), 7.17$ (d, J= $7.6 \mathrm{~Hz}, 1 \mathrm{H}), 6.93(\mathrm{~s}, 1 \mathrm{H}), 4.83(\mathrm{~s}$, $1 \mathrm{H}), 4.58(\mathrm{~s}, 1 \mathrm{H}), 4.39(\mathrm{~s}, 2 \mathrm{H}), 4.22(\mathrm{~s}, 2 \mathrm{H}), 3.34(\mathrm{~s}, 2 \mathrm{H}), 2.95(\mathrm{~s}, 3 \mathrm{H}) .{ }^{19} \mathrm{~F}\left\{{ }^{1} \mathrm{H}\right\}$ NMR $(377$ $\mathrm{MHz}): \delta-61.8$.

[1-(2-methoxymethyl-allyl)-1H-indol-3-ylmethylene]-phenyl-amine. This compound was prepared according to the general procedure for imine synthesis from $6(50.0 \mathrm{mg}, 0.218$ $\mathrm{mmol})$ and aniline $(22.3 \mathrm{mg}, 0.240 \mathrm{mmol})$ in $55 \%$ yield $(36.6 \mathrm{mg}) .{ }^{1} \mathrm{H}$ NMR (400 MHz, toluene$\left.d_{8}\right): \delta 8.92(\mathrm{~d}, J=7.7 \mathrm{~Hz}, 1 \mathrm{H}), 8.46(\mathrm{~s}, 1 \mathrm{H}), 7.31-6.96(\mathrm{~m}, 8 \mathrm{H}), 6.94(\mathrm{~s}, 1 \mathrm{H}), 4.85(\mathrm{~s}, 1 \mathrm{H}), 4.59$, $(\mathrm{s}, 1 \mathrm{H}), 4.23(\mathrm{~s}, 2 \mathrm{H}), 3.36(\mathrm{~s}, 2 \mathrm{H}), 2.95(\mathrm{~s}, 3 \mathrm{H})$.

4-Nitrobenzyl-[1-(2-methoxymethyl-allyl)-1H-indol-3-ylmethylene]-amine. This compound was prepared according to the general procedure for imine synthesis from 6 (50 $\mathrm{mg}$, $0.218 \mathrm{mmol})$ and 4-nitrobenzylamine $(36.5 \mathrm{mg}, 0.240 \mathrm{mmol})$. The product was isolated as a yellow solid in 74\% yield (58.6 mg). ${ }^{1} \mathrm{H}$ NMR (400 MHz, toluene- $\left.d_{8}\right): \delta 8.81(\mathrm{~d}, J=7.7 \mathrm{~Hz}$, $1 \mathrm{H}), 8.23(\mathrm{~s}, 1 \mathrm{H}), 7.85(\mathrm{~d}, J=8.4 \mathrm{~Hz}, 2 \mathrm{H}), 7.29(\mathrm{t}, J=7.6 \mathrm{~Hz}, 1 \mathrm{H}), 7.22(\mathrm{t}, J=7.7 \mathrm{~Hz}, 1 \mathrm{H}), 7.13$ 
(m, 3H), $6.92(\mathrm{~s}, 1 \mathrm{H}), 4.85(\mathrm{~s}, 1 \mathrm{H}), 4.60(\mathrm{~s}, 1 \mathrm{H}), 4.49(\mathrm{~s}, 2 \mathrm{H}), 4.25(\mathrm{~s}, 2 \mathrm{H}), 3.37(\mathrm{~s}, 2 \mathrm{H}), 2.96(\mathrm{~s}$, $3 \mathrm{H})$.

General Procedure for C-H Activation Reacions. $\left[\mathrm{RhCl}(\operatorname{coe})_{2}\right]_{2}(3.6 \mathrm{mg}, 0.0050$ mmol) and ligand $\mathbf{L}(5.4 \mathrm{mg}, 0.010 \mathrm{mmol})$ were premixed in toluene- $d_{8}(0.25 \mathrm{~mL})$ for approximately 5 min until all solid was dissolved. To this solution was added the imine substrate $(0.050 \mathrm{mmol})$ and 2,6-dimethoxytoluene (internal standard) dissolved in toluene- $d_{8}(0.25 \mathrm{~mL})$. The solution was heated to the appropriate temperature, and the reaction conversion relative to internal standard was monitored by ${ }^{1} \mathrm{H}$ NMR. Upon completion of the reaction, the cyclized imine was hydrolyzed by silica gel chromatography $\left(5 \% \mathrm{EtOAc} / \mathrm{CH}_{2} \mathrm{Cl}_{2}\right)$ and analyzed by chiral HPLC (CHIRALPAK AD column, 90/10 hexanes/EtOH, $1.0 \mathrm{~mL} / \mathrm{min}, \lambda=230 \mathrm{~nm}$ ) with (R)-7 eluting at $30.2 \mathrm{~min}$ and (S)-7 eluting at $40.9 \mathrm{~min}$.

(S)-2-Methoxymethyl-2,3-dihydro-1H-3a-aza-cyclopenta[a]indene-8-carboxaldehyde (S-7). To a stirred solution of fresh $\left[\mathrm{RhCl}(\text { coe })_{2}\right]_{2}(48.9 \mathrm{mg}, 0.0682 \mathrm{mmol})$ and ligand $\mathbf{L}$ ' (73.6 $\mathrm{mg}, 0.136 \mathrm{mmol})$ in toluene $(3.4 \mathrm{~mL})$ was added imine substrate 8 (310 $\mathrm{mg}, 0.682 \mathrm{mmol})$ dissolved in toluene $\left(6.8 \mathrm{~mL}, 0.1 \mathrm{M}\right.$ overall). The flask was sealed and heated to $90{ }^{\circ} \mathrm{C}$ for $21 \mathrm{~h}$, after which time the reaction mixture was concentrated, redissolved in $10 \% \mathrm{AcOH} / \mathrm{THF}(30$ $\mathrm{mL}$ ), and stirred for $6 \mathrm{~h}$ at room temperature. The mixture was concentrated, redissolved in $\mathrm{CH}_{2} \mathrm{Cl}_{2}$, and washed with water $(30 \mathrm{~mL})$, saturated aqueous sodium bicarbonate solution (30 $\mathrm{mL})$, and water $(30 \mathrm{~mL})$. The organic layer was then dried over magnesium sulfate, concentrated, and chromatographed $\left(5 \% \mathrm{EtOAc} / \mathrm{CH}_{2} \mathrm{Cl}_{2}\right)$ to provide the title compound in $61 \%$ yield $\left(95.2 \mathrm{mg}\right.$ ) and $90 \%$ ee. Mp: $87.9^{\circ} \mathrm{C}$. IR: $1641 \mathrm{~cm}^{-1} .{ }^{1} \mathrm{H}$ NMR $\left(400 \mathrm{MHz}, \mathrm{C}_{6} \mathrm{D}_{6}\right): \delta 10.19$ (s, 1H), $8.62(\mathrm{~m}, 1 \mathrm{H}), 7.24$ (t, $J=7.3 \mathrm{~Hz}, 1 \mathrm{H}), 7.13(\mathrm{~m}, 1 \mathrm{H}), 6.87$ (d, $J=8.1 \mathrm{~Hz}, 1 \mathrm{H}), 3.23$ (dd, $J$ $=8.2,10.6 \mathrm{~Hz}, 1 \mathrm{H}), 3.14(\mathrm{dd}, J=5.6,10.4 \mathrm{~Hz}, 1 \mathrm{H}), 2.92(\mathrm{~s}, 3 \mathrm{H}), 2.71(\mathrm{dd}, J=6.0,9.3 \mathrm{~Hz}, 1 \mathrm{H})$, $2.62(\mathrm{t}, J=8.4 \mathrm{~Hz}, 1 \mathrm{H}), 2.55(\mathrm{~m}, 1 \mathrm{H}), 2.42(\mathrm{~m}, 1 \mathrm{H}), 2.31(\mathrm{~m}, 1 \mathrm{H}) .{ }^{13} \mathrm{C}\left\{{ }^{1} \mathrm{H}\right\} \mathrm{NMR}(100 \mathrm{MHz}$, $\left.\mathrm{CDCl}_{3}\right): \delta 183.3,154.6,133.2,129.7,122.9,122.8,121.4,110.3,110.0,74.1,59.1,47.5,41.3$, 27.8. Anal. Calcd. for $\mathrm{C}_{14} \mathrm{H}_{15} \mathrm{NO}_{2}$ : C, 73.34; H, 6.59; N, 6.11. Found: C, 73.11; H, 6.61; N, 6.02. $[\alpha]_{\mathrm{D}}^{25}:-9.57\left(c=1.17, \mathrm{CH}_{2} \mathrm{Cl}_{2}\right), 90 \%$ ee.

\section{(S)- $N$-(4-Bromo-2-nitrophenyl)- $N^{\prime}$-(2-methoxymethyl-2,3-dihydro-1 $H$-3a-aza-}

cyclopenta $[a]$ inden-8-ylmethylene)-hydrazine (12). To a solution of 4-bromo-2nitrophenylhydrazine $(64.2 \mathrm{mg}, 0.277 \mathrm{mmol})$ in ethanol $(9.6 \mathrm{~mL}, 0.029 \mathrm{M})$ and concentrated sulfuric acid (3 drops) was added 7 (90\% ee, 63.4mg, $0.277 \mathrm{mmol})$ dissolved in a minimum 
amount of 2:1 ethanol/water. The solution was stirred overnight (12 h) at room temperature, after which time it was basified with $2 \mathrm{M}$ sodium carbonate and extracted with $\mathrm{CH}_{2} \mathrm{Cl}_{2}(3 \times 10$ $\mathrm{mL}$ ). The organic extracts were dried over magnesium sulfate, concentrated, and chromatographed on silica (EtOAc) to provide the title compound as a purple solid in $82 \%$ yield (100 mg). X-ray quality crystals were subsequently grown by vapor diffusion at $-35{ }^{\circ} \mathrm{C}$ $\left(\mathrm{CH}_{2} \mathrm{Cl}_{2} /\right.$ pentane $)$, and the structure obtained shows an (S)-configuration by anomalous dispersion (see next section). ${ }^{1} \mathrm{H}$ NMR (400 MHz, $\left.\mathrm{C}_{6} \mathrm{D}_{6}\right): \delta 10.83(\mathrm{~s}, 1 \mathrm{H}), 8.46(\mathrm{~d}, J=8.1 \mathrm{~Hz}$, $1 \mathrm{H}), 8.29(\mathrm{~d}, J=2.3,1 \mathrm{H}), 7.79(\mathrm{~d}, J=9.3,1 \mathrm{H}), 7.65(\mathrm{~s}, 1 \mathrm{H}), 7.39(\mathrm{t}, J=7.7 \mathrm{~Hz}, 1 \mathrm{H}), 7.26(\mathrm{t}, J=$ $9.3 \mathrm{~Hz}, 1 \mathrm{H}), 7.04(\mathrm{~m}, 2 \mathrm{H}), 3.41$ (dd, $J=7.5,10.3 \mathrm{~Hz}, 1 \mathrm{H}), 3.27$ (m, 1H), 3.00 (s, 3H), 2.87 (dd, $J$ $=6.0,9.1 \mathrm{~Hz}, 1 \mathrm{H}), 2.81(\mathrm{~m}, 1 \mathrm{H}), 2.60(\mathrm{~m}, 2 \mathrm{H}), 2.40(\mathrm{~m}, 1 \mathrm{H})$.

(S)-2-Methoxymethyl-2,3-dihydro-1H-3a-aza-cyclopenta[a]indene (9). This compound was prepared according to the general procedure for the catalytic decarbonylation of indole-2-carboxaldehydes published by Meyer and $\mathrm{Kruse}^{4}$ with $5 \%$ rhodium catalyst and was isolated as a pale yellow oil in $86 \%$ yield $(53.0 \mathrm{mg}) .{ }^{1} \mathrm{H} \mathrm{NMR}\left(400 \mathrm{MHz}, \mathrm{CDCl}_{3}\right): \delta 7.54(\mathrm{~d}, J=$ $8.2 \mathrm{~Hz}, 1 \mathrm{H}), 7.22$ (d, $J=7.8 \mathrm{~Hz}, 1 \mathrm{H}), 7.11$ (t, $J=7.0 \mathrm{~Hz}, 1 \mathrm{H}), 7.05$ (t, $J=6.9 \mathrm{~Hz}, 1 \mathrm{H}), 4.18$ (dd, $J=7.7,10.2 \mathrm{~Hz}, 1 \mathrm{H}), 3.89(\mathrm{dd}, J=5.4,10.4 \mathrm{~Hz}, 1 \mathrm{H}), 3.51(\mathrm{dd}, J=6.4,9.1 \mathrm{~Hz}, 1 \mathrm{H}), 3.44(\mathrm{t}, J=$ $8.0 \mathrm{~Hz}, 1 \mathrm{H}), 3.38(\mathrm{~s}, 3 \mathrm{H}), 3.27(\mathrm{~m}, 1 \mathrm{H}), 3.14(\mathrm{dd}, J=8.3,15.9 \mathrm{~Hz}, 1 \mathrm{H}), 2.78(\mathrm{dd}, J=5.5,15.9$ $\mathrm{Hz}, 1 \mathrm{H}) .{ }^{13} \mathrm{C}\left\{{ }^{1} \mathrm{H}\right\} \mathrm{NMR}(100 \mathrm{MHz}): \delta 143.3,133.0,132.7,120.3,120.2,119.1,109.3,92.7$, 75.0, 59.0, 46.7, 42.1, 27.6. EI-HRMS Calcd. for $\mathrm{C}_{13} \mathrm{H}_{15} \mathrm{NO}[\mathrm{M}]^{+}:$201.1154. Found: 201.1150.

3,4-Dibromo-1-(2,4-dimethoxybenzyl)pyrrole-2,5-dione (10). ${ }^{5}$ To a round bottom flask was added 2,3-dibromomaleic acid (0.660 g, $2.41 \mathrm{mmol})$, DCC (0.520 g, $2.53 \mathrm{mmol})$, and $\mathrm{CH}_{2} \mathrm{Cl}_{2}(25 \mathrm{~mL}, 0.10 \mathrm{M})$, and the resulting solution was stirred for $1 \mathrm{~h}$ at room temperature. After this time, the urea produced during the reaction was removed by filtration, and 2,4dimethoxybenzylamine was added $(0.403 \mathrm{~g}, 2.41 \mathrm{mmol})$. The resulting solution was stirred for 1 $\mathrm{h}$ at room temperature, after which time it was concentrated, redissolved in $\mathrm{AcOH}(20 \mathrm{~mL})$, and heated to reflux overnight. After the reaction mixture was cooled to room temperature, it was concentrated, redissolved in $\mathrm{CH}_{2} \mathrm{Cl}_{2}$, and washed with water $(20 \mathrm{~mL}), 2 \mathrm{M}$ sodium carbonate (20 $\mathrm{mL})$, and water $(20 \mathrm{~mL})$, dried over magnesium sulfate, concentrated, and chromatographed on silica (50\% $\mathrm{CH}_{2} \mathrm{Cl}_{2} /$ hexanes) to afford the title compound as pale yellow crystals in $43 \%$ yield (0.420 g). Mp: $130-132{ }^{\circ} \mathrm{C}$. IR: $1717 \mathrm{~cm}^{-1} .{ }^{1} \mathrm{H}$ NMR $\left(500 \mathrm{MHz}, \mathrm{C}_{6} \mathrm{D}_{6}\right): \delta 7.22(\mathrm{~d}, J=8.5 \mathrm{~Hz}$, $1 \mathrm{H}), 6.30(\mathrm{~d}, J=2.6 \mathrm{~Hz}, 1 \mathrm{H}), 6.24(\mathrm{dd}, J=2.4,8.4 \mathrm{~Hz}, 1 \mathrm{H}), 4.56$ (s, 2H), 3.28 (s, 3H), 3.22 (s, 
$3 \mathrm{H}) .{ }^{13} \mathrm{C}\left\{{ }^{1} \mathrm{H}\right\}$ NMR $\left(125 \mathrm{MHz}, \mathrm{CDCl}_{3}\right): \delta 163.5,160.9,158.3,131.0,129.3,115.5,103.9,98.4$, 55.4, 55.3, 38.5. Anal. Calcd. for $\mathrm{C}_{13} \mathrm{H}_{11} \mathrm{Br}_{2} \mathrm{NO}_{4}$ : C, 38.55; H, 2.74; N, 3.46. Found: $\mathrm{C}, 38.61$; $\mathrm{H}, 2.58 ; \mathrm{N}, 3.52$.

(S)-3-Bromo-1-(2,4-dimethoxy-benzyl)-4-(2-methoxymethyl-2,3-dihydro-1H-3a-azacyclopenta $[a]$ inden-8-yl)-pyrrole-2,5-dione (11). In an inert atmosphere glove box, a glass reaction vessel equipped with a vacuum stopcock and a stir bar was charged with 9 (39.0 mg, $0.194 \mathrm{mmol}), 10$ (157 mg, $0.388 \mathrm{mmol})$, potassium carbonate $(52.3 \mathrm{mg}, 0.388 \mathrm{mmol})$ and THF $(6.0 \mathrm{~mL}, 0.032 \mathrm{M})$. The flask was sealed and the reaction mixture was heated to $85^{\circ} \mathrm{C}$ for $5.5 \mathrm{~d}$, after which time it was concentrated and chromatographed (40\% EtOAc/hexanes) to afford the title compound as an orange foam in $75 \%$ yield $(76.0 \mathrm{mg})$. IR: $1709 \mathrm{~cm}^{-1} .{ }^{1} \mathrm{H}$ NMR $(500 \mathrm{MHz}$, $\left.\mathrm{CDCl}_{3}\right): \delta 7.66(\mathrm{~d}, J=8.1 \mathrm{~Hz}, 1 \mathrm{H}), 7.28-7.16(\mathrm{~m}, 4 \mathrm{H}), 6.44(\mathrm{~m}, 2 \mathrm{H}), 4.79(\mathrm{~s}, 2 \mathrm{H}), 4.26(\mathrm{dd}, J=$ 7.8, $10.4 \mathrm{~Hz}, 1 \mathrm{H}), 4.02$ (dd, $J=5.2,10.7 \mathrm{~Hz}, 1 \mathrm{H}), 3.83$ (s, 3H), 3.79 (s, $3 \mathrm{H}), 3.55$ (dd, $J=5.3$, $9.0 \mathrm{~Hz}, 1 \mathrm{H}), 3.46$ (t, $J=8.7 \mathrm{~Hz}, 1 \mathrm{H}), 3.39$ (s, $3 \mathrm{H}), 3.33$ (m, 2H), 3.03 (dd, $J=4.9,16.3 \mathrm{~Hz}, 1 \mathrm{H})$. ${ }^{13} \mathrm{C}\left\{{ }^{1} \mathrm{H}\right\}$ NMR (125 MHz): $\delta 169.0,166.4,160.5,158.2,147.4,138.6,133.2,130.3,129.7$, 122.2, 121.9, 120.7, 116.6, 115.4, 110.1, 103.9, 98.4, 96.9, 74.4, 59.0, 55.4, 55.3, 47.6, 41.5, 37.4, 30.0. FAB-HRMS Calcd. for $\mathrm{C}_{26} \mathrm{H}_{25} \mathrm{BrN}_{2} \mathrm{O}_{5}[\mathrm{M}]^{+}: 524.0947$. Found: 524.0938. [ $\left.\alpha\right]_{\mathrm{D}}^{25}$ : $40.25\left(c=1.18, \mathrm{CH}_{2} \mathrm{Cl}_{2}\right), 90 \%$ ee.

\section{(S)-1-(2,4-Dimethoxy-benzyl)-3-(2-methoxymethyl-2,3-dihydro-1 H-3a-aza-} cyclopenta $[a]$ inden-8-yl)-4-phenylamino-pyrrole-2,5-dione (15). ${ }^{6}$ To a premixed solution of palladium acetate $(0.14 \mathrm{mg}, 0.00062 \mathrm{mmol})$ and (R)-BINAP $(0.58 \mathrm{mg}, 0.00093 \mathrm{mmol})$ in toluene $(0.1 \mathrm{~mL})$ was added $11(6.5 \mathrm{mg}, 0.012 \mathrm{mmol})$, cesium carbonate $(5.6 \mathrm{mg}, 0.017 \mathrm{mmol})$, and aniline $(1.4 \mathrm{mg}, 0.015 \mathrm{mmol})$ dissolved in toluene $(0.45 \mathrm{~mL})$. The resulting light orange solution was heated for $14 \mathrm{~h}$ at $105^{\circ} \mathrm{C}$, at which point it had become a reddish-orange. The solution was concentrated and chromatographed (10:30:60 $\mathrm{Et}_{3} \mathrm{~N}$ :EtOAc:hexanes) to afford the title compound as an reddish-orange foam in 62\% yield (4.1 mg). IR: $3307,1696 \mathrm{~cm}^{-1} .{ }^{1} \mathrm{H}$ NMR (400 MHz, $\left.\mathrm{C}_{6} \mathrm{D}_{6}\right): \delta 7.77(\mathrm{~d}, J=7.3 \mathrm{~Hz}, 1 \mathrm{H}), 7.53(\mathrm{~d}, J=8.3 \mathrm{~Hz}, 1 \mathrm{H}), 7.10(\mathrm{~m}, 2 \mathrm{H}), 6.96(\mathrm{~m}, 1 \mathrm{H}), 6.95(\mathrm{~s}$, 1H), 6.55 (t, $J=8.1 \mathrm{~Hz}, 2 \mathrm{H}), 6.46$ (t, $J=7.3 \mathrm{~Hz}, 1 \mathrm{H}), 6.39$ (d, $J=2.5 \mathrm{~Hz}, 1 \mathrm{H}), 6.32(\mathrm{~m}, 3 \mathrm{H})$, 5.04 (s, 2H), $3.42(\mathrm{dd}, J=7.8,10.4 \mathrm{~Hz}, 1 \mathrm{H}), 3.32(\mathrm{~s}, 3 \mathrm{H}), 3.31(\mathrm{~m}, 1 \mathrm{H}), 3.30(\mathrm{~s}, 3 \mathrm{H}), 2.93$ (s, $3 \mathrm{H}), 2.72(\mathrm{dd}, J=5.6,8.8 \mathrm{~Hz}, 1 \mathrm{H}), 2.64-2.44(\mathrm{~m}, 3 \mathrm{H}), 2.22(\mathrm{dd}, J=4.6,15.7 \mathrm{~Hz}, 1 \mathrm{H}) .{ }^{13} \mathrm{C}\left\{{ }^{1} \mathrm{H}\right\}$ NMR (125 MHz, $\left.\mathrm{CDCl}_{3}\right): \delta 171.9,169.1,160.4,158.1,145.0,137.0,134.0,132.7,131.5,130.0$, $127.8,123.1,120.8,120.7,119.8,119.7,117.2,109.2,103.9,100.5,98.4,97.4,74.6,58.8,55.5$, 
55.3, 46.9, 41.2, 36.5, 27.8. FAB-HRMS Calcd. for $\mathrm{C}_{32} \mathrm{H}_{31} \mathrm{~N}_{3} \mathrm{O}_{5}[\mathrm{M}]^{+}:$537.2264. Found: 537.2269. $[\alpha]_{\mathrm{D}}^{25}:-20.45\left(c=0.88, \mathrm{CH}_{2} \mathrm{Cl}_{2}\right), 90 \%$ ee.

(S)-3-(2-Methoxymethyl-2,3-dihydro-1H-3a-aza-cyclopenta[a]inden-8-yl)-4-

phenylamino-pyrrole-2,5-dione (2). ${ }^{5} \quad$ A solution of 15 (7.5 $\left.\mathrm{mg}, 0.019 \mathrm{mmol}\right)$ and methanesulfonic acid $(1.0 \mathrm{~mL})$ in $\mathrm{CH}_{2} \mathrm{Cl}_{2}(2.0 \mathrm{~mL})$ was stirred for $30 \mathrm{~min}$ at room temperature, after which it was cooled to $0{ }^{\circ} \mathrm{C}$, quenched slowly with ammonium hydroxide, and extracted with $\mathrm{CH}_{2} \mathrm{Cl}_{2}(3 \times 5 \mathrm{~mL})$. The combined organic layers were washed with water and brine, dried over magnesium sulfate, concentrated, and chromatographed $\left(20 \% \mathrm{Et}_{2} \mathrm{O} / \mathrm{CH}_{2} \mathrm{Cl}_{2}\right)$ to provide the title compound as an orange solid in $61 \%$ yield $(3.3 \mathrm{mg})$. IR: $3304,1705 \mathrm{~cm}^{-1}$. ${ }^{1} \mathrm{H}$ NMR (500 $\left.\mathrm{MHz}, \mathrm{CDCl}_{3}\right): \delta 7.50(\mathrm{~d}, J=7.9 \mathrm{~Hz}, 1 \mathrm{H}), 7.33(\mathrm{~s}, 1 \mathrm{H}), 7.12(\mathrm{~m}, 3 \mathrm{H}), 7.04(\mathrm{t}, J=7.8 \mathrm{~Hz}, 1 \mathrm{H})$, $6.90(\mathrm{t}, J=7.8 \mathrm{~Hz}, 2 \mathrm{H}), 6.85(\mathrm{t}, J=7.2 \mathrm{~Hz}, 1 \mathrm{H}), 6.64$ (d, $J=7.9 \mathrm{~Hz}, 2 \mathrm{H}), 4.02(\mathrm{dd}, J=7.5,10.2$ $\mathrm{Hz}, 1 \mathrm{H}), 3.78(\mathrm{~m}, 1 \mathrm{H}), 3.29(\mathrm{~s}, 3 \mathrm{H}), 3.14(\mathrm{~m}, 1 \mathrm{H}), 2.98(\mathrm{~m}, 1 \mathrm{H}), 2.91(\mathrm{~m}, 1 \mathrm{H}), 2.63(\mathrm{~m}, 1 \mathrm{H})$, $2.20(\mathrm{dd}, J=4.9,16.8 \mathrm{~Hz}, 1 \mathrm{H}) .{ }^{13} \mathrm{C}\left\{{ }^{1} \mathrm{H}\right\} \mathrm{NMR}(125 \mathrm{MHz}): \delta 171.4,168.8,145.2,136.6,134.7$, 132.7, 131.3, 127.8, 123.5, 121.0, 120.5, 120.0, 119.9, 109.4, 101.7, 96.9, 74.5, 58.8, 46.9, 41.2, 27.7. FAB-HRMS Calcd. for $\mathrm{C}_{23} \mathrm{H}_{21} \mathrm{~N}_{3} \mathrm{O}_{3}[\mathrm{M}]^{+}: 387.1583$. Found: 387.1575 . $[\alpha]_{\mathrm{D}}^{25}:-4.55(c=$ $\left.0.11, \mathrm{CH}_{2} \mathrm{Cl}_{2}\right), 90 \%$ ee. 


\section{Structural Data for 12.}

Figure S-1. ORTEP Diagram of 12.

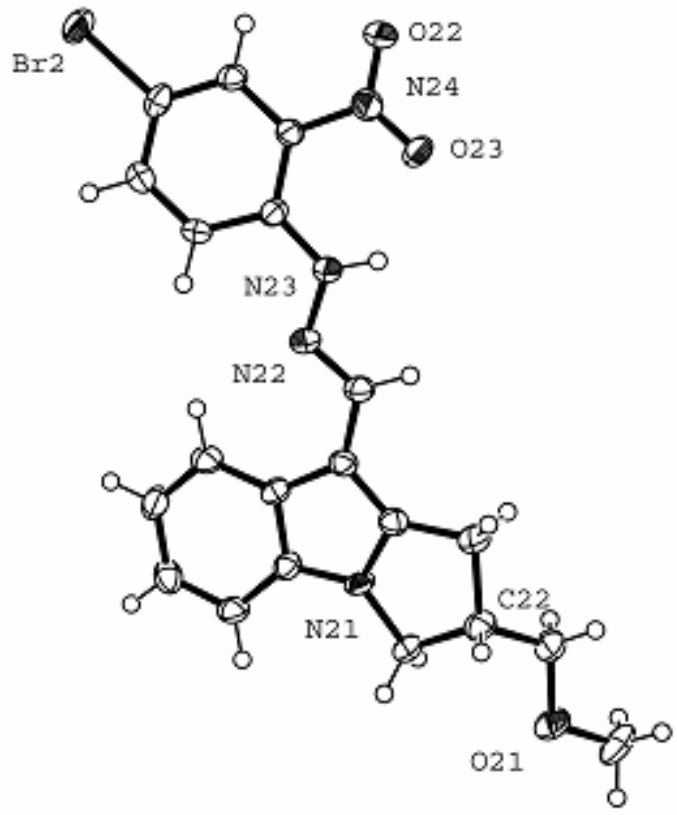

Table S-1. Crystal and Data Collection Parameters.

\section{A. Crystal Data \\ Empirical Formula \\ Formula Weight \\ Crystal Color, Habit \\ Crystal Dimensions \\ Crystal System \\ Lattice Type \\ Lattice Parameters}

$$
\begin{aligned}
& \mathrm{C}_{20} \mathrm{H}_{19} \mathrm{BrN}_{4} \mathrm{O}_{3} \\
& 443.30 \\
& \text { dark purple, plate } \\
& 0.17 \text { x } 0.16 \times 0.02 \mathrm{~mm} \\
& \text { monoclinic } \\
& \text { primitive } \\
& \mathrm{a}=8.481(1) \AA \\
& \mathrm{b}=10.202(1) \AA \\
& \mathrm{c}=21.606(3) \AA \\
& \alpha=90^{\circ} \\
& \beta=97.851(4)^{\circ} \\
& \gamma=90^{\circ} \\
& \mathrm{V}=1852.1(4) \AA^{3} \\
& \mathrm{P} 21 \\
& 4 \\
& 1.590 \mathrm{~g} / \mathrm{cm}^{3} \\
& 904 \\
& 2.77 \mathrm{~cm}^{-1}
\end{aligned}
$$

$\mathrm{F}_{000}$ 
B. Intensity Measurements Diffractometer

Radiation

monochromated

Detector Position

Exposure Time

Scan Type

$\theta_{\max }$

No. of Reflections Measured

Corrections

C. Structure Solution and Refinement Structure Solution

Refinement

Function Minimized

Least Squares Weighting scheme

q-factor

Anomalous Dispersion

No. Observations $(\mathrm{I}>2.00 \sigma(\mathrm{I}))$

No. Variables

Reflection/Parameter Ratio

Residuals: R; $\mathrm{wR}_{2} ; \mathrm{R}_{\text {all }}$

Goodness of Fit Indicator

Max Shift/Error in Final Cycle

Maximum peak in Final Diff. Map

Minimum peak in Final Diff. Map
Bruker Platinum 200

synchrotron $(\lambda=0.77500 \AA)$

$\mathrm{Si}-<111>$ channel cut crystal

$74.00 \mathrm{~mm}$

2 seconds per frame.

$\omega$ (0.3 degrees per frame)

$29.05^{\circ}$

Total: 23162

Unique: $7432\left(\mathrm{R}_{\text {int }}=0.0643\right)$

Lorentz-polarization

Absorption $\left(\mathrm{T}_{\max }=0.9466\right.$,

$\mathrm{T}_{\min }=0.6500$ )

direct (SHELXS-97 (Sheldrick, 1990))

Full-matrix least-squares

$\Sigma \omega\left(|\mathrm{Fo}|^{2}-|\mathrm{Fc}|^{2}\right)^{2}$

$\omega=1 /\left[\sigma^{2}\left(\mathrm{Fo}^{2}\right)+(\mathrm{qP})^{2}+0.000 \mathrm{P}\right]$

where $\mathrm{P}=\left[\mathrm{Fo}^{2}+2 \mathrm{Fc}^{2}\right] / 3$

0.0106

All non-hydrogen atoms

5893

508

11.60

$0.0350 ; 0.0678 ; 0.0431$

0.922

0.002

$0.260 \mathrm{e}-/ \AA^{3}$

$-0.398 \mathrm{e}-/ \AA^{3}$ 
Table S-2. Atomic Coordinates and Uiso/Ueq and Occupancy

\begin{tabular}{|c|c|c|c|c|c|}
\hline atom & $\mathrm{X}$ & $\mathrm{y}$ & $\mathrm{z}$ & Ueq & Occupancy \\
\hline $\mathrm{Br} 1$ & $-0.1935(1)$ & $0.2191(1)$ & $-0.0500(1)$ & $0.044(1)$ & 1 \\
\hline $\mathrm{Br} 2$ & $0.6768(1)$ & $0.7422(1)$ & $0.5539(1)$ & $0.041(1)$ & 1 \\
\hline $\mathrm{O} 1$ & $1.0580(3)$ & $0.0099(3)$ & $0.5095(1)$ & $0.048(1)$ & 1 \\
\hline $\mathrm{O} 2$ & $-0.0640(3)$ & $0.5376(3)$ & $0.1403(1)$ & $0.045(1)$ & 1 \\
\hline $\mathrm{O} 3$ & $0.1572(3)$ & $0.4840(3)$ & $0.1968(1)$ & $0.039(1)$ & 1 \\
\hline $\mathrm{O} 21$ & $-0.5123(3)$ & $0.9569(3)$ & $-0.0177(1)$ & $0.040(1)$ & 1 \\
\hline $\mathrm{O} 22$ & $0.5740(3)$ & $0.4202(2)$ & $0.3617(1)$ & $0.038(1)$ & 1 \\
\hline $\mathrm{O} 23$ & $0.3417(3)$ & $0.4588(3)$ & $0.3093(1)$ & $0.039(1)$ & 1 \\
\hline N1 & $0.7043(3)$ & $-0.0924(3)$ & $0.3505(1)$ & $0.028(1)$ & 1 \\
\hline N2 & $0.3490(4)$ & $0.1310(3)$ & $0.2094(1)$ & $0.026(1)$ & 1 \\
\hline N3 & $0.2670(3)$ & $0.2443(3)$ & $0.1901(1)$ & $0.028(1)$ & 1 \\
\hline N4 & $0.0477(4)$ & $0.4617(3)$ & $0.1528(1)$ & $0.030(1)$ & 1 \\
\hline N21 & $-0.2301(3)$ & $1.0325(3)$ & $0.1585(1)$ & $0.026(1)$ & 1 \\
\hline N22 & $0.1295(4)$ & $0.8078(3)$ & $0.2970(1)$ & $0.028(1)$ & 1 \\
\hline $\mathrm{N} 23$ & $0.2132(4)$ & $0.6943(3)$ & $0.3151(1)$ & $0.030(1)$ & 1 \\
\hline N24 & $0.4525(4)$ & $0.4879(3)$ & $0.3503(1)$ & $0.032(1)$ & 1 \\
\hline $\mathrm{C} 1$ & $0.8329(4)$ & $-0.0995(4)$ & $0.4022(2)$ & $0.033(1)$ & 1 \\
\hline $\mathrm{C} 2$ & $0.8041(4)$ & $0.0272(3)$ & $0.4392(1)$ & $0.031(1)$ & 1 \\
\hline $\mathrm{C} 3$ & $0.7067(4)$ & $0.1187(4)$ & $0.3916(2)$ & $0.032(1)$ & 1 \\
\hline $\mathrm{C} 4$ & $0.6338(4)$ & $0.0267(4)$ & $0.3415(2)$ & $0.029(1)$ & 1 \\
\hline $\mathrm{C} 5$ & $0.5232(4)$ & $0.0266(4)$ & $0.2888(2)$ & $0.024(1)$ & 1 \\
\hline C6 & $0.5273(4)$ & $-0.1051(3)$ & $0.2633(2)$ & $0.025(1)$ & 1 \\
\hline $\mathrm{C} 7$ & $0.4419(5)$ & $-0.1709(4)$ & $0.2134(2)$ & $0.030(1)$ & 1 \\
\hline $\mathrm{C} 8$ & $0.4740(5)$ & $-0.2991(4)$ & $0.2023(2)$ & $0.038(1)$ & 1 \\
\hline C9 & $0.5958(5)$ & $-0.3669(4)$ & $0.2411(2)$ & $0.039(1)$ & 1 \\
\hline $\mathrm{C} 10$ & $0.6820(5)$ & $-0.3043(4)$ & $0.2912(2)$ & $0.033(1)$ & 1 \\
\hline C11 & $0.6460(4)$ & $-0.1766(4)$ & $0.3024(2)$ & $0.026(1)$ & 1 \\
\hline C12 & $0.9561(3)$ & $0.0907(3)$ & $0.4680(1)$ & $0.043(1)$ & 1 \\
\hline C13 & $1.0128(3)$ & $0.0057(3)$ & $0.5697(1)$ & $0.052(1)$ & 1 \\
\hline C14 & $0.4331(4)$ & $0.1367(3)$ & $0.2629(2)$ & $0.027(1)$ & 1 \\
\hline $\mathrm{C} 15$ & $0.1620(4)$ & $0.2423(4)$ & $0.1375(1)$ & $0.024(1)$ & 1 \\
\hline C16 & $0.0560(4)$ & $0.3441(3)$ & $0.1175(2)$ & $0.024(1)$ & 1 \\
\hline $\mathrm{C} 17$ & $-0.0537(4)$ & $0.3366(4)$ & $0.0632(2)$ & $0.028(1)$ & 1 \\
\hline C18 & $-0.0541(4)$ & $0.2289(5)$ & $0.0259(2)$ & $0.032(1)$ & 1 \\
\hline C19 & $0.0487(5)$ & $0.1256(4)$ & $0.0446(2)$ & $0.040(1)$ & 1 \\
\hline $\mathrm{C} 20$ & $0.1512(5)$ & $0.1305(4)$ & $0.0983(2)$ & $0.035(1)$ & 1 \\
\hline $\mathrm{C} 21$ & $-0.3518(4)$ & $1.0421(4)$ & $0.1042(2)$ & $0.030(1)$ & 1 \\
\hline $\mathrm{C} 22$ & $-0.3700(4)$ & $0.8988(3)$ & $0.0812(1)$ & $0.031(1)$ & 1 \\
\hline $\mathrm{C} 23$ & $-0.2263(4)$ & $0.8221(4)$ & $0.1157(2)$ & $0.035(1)$ & 1 \\
\hline $\mathrm{C} 24$ & $-0.1590(4)$ & $0.9127(3)$ & $0.1666(2)$ & $0.027(1)$ & 1 \\
\hline $\mathrm{C} 25$ & $-0.0471(4)$ & $0.9142(3)$ & $0.2190(2)$ & $0.025(1)$ & 1 \\
\hline $\mathrm{C} 26$ & $-0.0477(4)$ & $1.0445(3)$ & $0.2431(2)$ & $0.024(1)$ & 1 \\
\hline
\end{tabular}




$\begin{array}{llllll}\text { C27 } & 0.0435(5) & 1.1106(4) & 0.2929(2) & 0.030(1) & 1 \\ \text { C28 } & 0.0139(4) & 1.2419(5) & 0.3018(2) & 0.036(1) & 1 \\ \text { C29 } & -0.1069(5) & 1.3080(4) & 0.2645(2) & 0.035(1) & 1 \\ \text { C30 } & -0.1975(4) & 1.2487(4) & 0.2151(2) & 0.032(1) & 1 \\ \text { C31 } & -0.1639(4) & 1.1156(4) & 0.2051(2) & 0.026(1) & 1 \\ \text { C32 } & -0.3802(4) & 0.8845(3) & 0.0116(1) & 0.037(1) & 1 \\ \text { C33 } & -0.5296(5) & 0.9424(4) & -0.0835(2) & 0.047(1) & 1 \\ \text { C34 } & 0.0445(4) & 0.8016(3) & 0.2433(2) & 0.027(1) & 1 \\ \text { C35 } & 0.3197(4) & 0.6991(3) & 0.3685(2) & 0.024(1) & 1 \\ \text { C36 } & 0.4377(4) & 0.6029(3) & 0.3869(2) & 0.024(1) & 1 \\ \text { C37 } & 0.5473(4) & 0.6187(3) & 0.4404(2) & 0.026(1) & 1 \\ \text { C38 } & 0.5353(4) & 0.7242(5) & 0.4779(2) & 0.030(1) & 1 \\ \text { C39 } & 0.4189(5) & 0.8174(4) & 0.4625(2) & 0.041(1) & 1 \\ \text { C40 } & 0.3166(5) & 0.8066(4) & 0.4089(2) & 0.035(1) & 1\end{array}$

Ueq is defined as one third of the orthogonalized Uij tensor 
Table S-3. Anisotropic Displacement Parameters

\begin{tabular}{|c|c|c|c|c|c|c|}
\hline atom & U11 & U22 & U33 & U12 & U13 & $\mathrm{U} 23$ \\
\hline $\mathrm{Br} 1$ & $0.045(1)$ & $0.054(1)$ & $0.030(1)$ & $-0.006(1)$ & $-0.011(1)$ & $0.001(1)$ \\
\hline $\mathrm{Br} 2$ & $0.031(1)$ & $0.058(1)$ & $0.030(1)$ & $-0.010(1)$ & $-0.007(1)$ & $0.002(1)$ \\
\hline $\mathrm{O} 1$ & $0.041(2)$ & $0.064(2)$ & $0.034(2)$ & $0.012(1)$ & $-0.009(1)$ & $0.001(1)$ \\
\hline $\mathrm{O} 2$ & $0.047(2)$ & $0.030(2)$ & $0.052(2)$ & $-0.006(1)$ & $-0.011(2)$ & $0.017(1)$ \\
\hline $\mathrm{O} 3$ & $0.047(2)$ & $0.030(2)$ & $0.035(2)$ & $-0.006(1)$ & $-0.012(1)$ & $0.004(1)$ \\
\hline $\mathrm{O} 21$ & $0.043(2)$ & $0.047(2)$ & $0.027(2)$ & $0.006(1)$ & $-0.008(1)$ & $0.006(1)$ \\
\hline $\mathrm{O} 22$ & $0.040(2)$ & $0.029(2)$ & $0.041(2)$ & $-0.002(1)$ & $-0.007(1)$ & $0.012(1)$ \\
\hline $\mathrm{O} 23$ & $0.048(2)$ & $0.029(2)$ & $0.032(2)$ & $-0.005(1)$ & $-0.018(1)$ & $0.005(1)$ \\
\hline N1 & $0.030(2)$ & $0.029(2)$ & $0.025(2)$ & $0.003(1)$ & $0.001(1)$ & $0.006(1)$ \\
\hline N2 & $0.025(2)$ & $0.025(2)$ & $0.027(2)$ & $0.004(1)$ & $0.002(1)$ & $0.007(1)$ \\
\hline N3 & $0.038(2)$ & $0.021(2)$ & $0.023(1)$ & $-0.002(1)$ & $-0.002(1)$ & $0.007(2)$ \\
\hline N4 & $0.038(2)$ & $0.023(2)$ & $0.026(2)$ & $0.007(1)$ & $-0.005(2)$ & $0.003(2)$ \\
\hline $\mathrm{N} 21$ & $0.023(2)$ & $0.033(2)$ & $0.021(2)$ & $0.005(1)$ & $-0.004(1)$ & $0.005(1)$ \\
\hline $\mathrm{N} 22$ & $0.028(2)$ & $0.028(2)$ & $0.026(2)$ & $0.004(1)$ & $-0.001(1)$ & $0.005(1)$ \\
\hline $\mathrm{N} 23$ & $0.033(2)$ & $0.025(2)$ & $0.029(2)$ & $-0.001(1)$ & $-0.005(1)$ & $0.009(1)$ \\
\hline N24 & $0.042(2)$ & $0.022(2)$ & $0.032(2)$ & $0.002(1)$ & $0.001(2)$ & $0.002(2)$ \\
\hline $\mathrm{C} 1$ & $0.024(2)$ & $0.043(2)$ & $0.029(2)$ & $0.009(2)$ & $-0.002(2)$ & $0.005(2)$ \\
\hline $\mathrm{C} 2$ & $0.030(2)$ & $0.041(2)$ & $0.021(2)$ & $0.007(2)$ & $0.000(2)$ & $-0.003(2)$ \\
\hline $\mathrm{C} 3$ & $0.036(2)$ & $0.032(2)$ & $0.027(2)$ & $0.002(2)$ & $-0.002(2)$ & $0.004(2)$ \\
\hline $\mathrm{C} 4$ & $0.029(2)$ & $0.033(2)$ & $0.025(2)$ & $0.004(2)$ & $0.008(2)$ & $0.003(2)$ \\
\hline $\mathrm{C} 5$ & $0.023(2)$ & $0.028(2)$ & $0.022(2)$ & $0.004(1)$ & $0.003(2)$ & $0.003(2)$ \\
\hline C6 & $0.026(2)$ & $0.027(2)$ & $0.023(2)$ & $0.002(2)$ & $0.007(2)$ & $0.002(2)$ \\
\hline $\mathrm{C} 7$ & $0.033(2)$ & $0.034(2)$ & $0.022(2)$ & $0.001(2)$ & $0.001(2)$ & $0.002(2)$ \\
\hline $\mathrm{C} 8$ & $0.054(3)$ & $0.035(3)$ & $0.025(2)$ & $-0.001(2)$ & $0.004(2)$ & $0.005(2)$ \\
\hline C9 & $0.054(3)$ & $0.027(2)$ & $0.036(2)$ & $0.002(2)$ & $0.004(2)$ & $0.008(2)$ \\
\hline C10 & $0.037(2)$ & $0.034(2)$ & $0.028(2)$ & $0.005(2)$ & $0.005(2)$ & $0.008(2)$ \\
\hline C11 & $0.028(2)$ & $0.031(2)$ & $0.020(2)$ & $0.005(2)$ & $0.005(2)$ & $0.004(2)$ \\
\hline C12 & $0.041(2)$ & $0.057(2)$ & $0.028(2)$ & $0.011(2)$ & $-0.007(2)$ & $-0.004(2)$ \\
\hline C13 & $0.054(3)$ & $0.067(3)$ & $0.029(2)$ & $0.010(2)$ & $-0.020(2)$ & $-0.016(2)$ \\
\hline C14 & $0.033(2)$ & $0.025(2)$ & $0.023(2)$ & $0.001(1)$ & $0.006(2)$ & $-0.001(2)$ \\
\hline C15 & $0.028(2)$ & $0.023(2)$ & $0.020(2)$ & $0.004(2)$ & $0.001(1)$ & $-0.001(2)$ \\
\hline C16 & $0.031(2)$ & $0.021(2)$ & $0.020(2)$ & $0.003(1)$ & $0.002(2)$ & $0.000(2)$ \\
\hline C17 & $0.028(2)$ & $0.030(2)$ & $0.024(2)$ & $0.007(2)$ & $-0.002(2)$ & $-0.001(2)$ \\
\hline C18 & $0.027(2)$ & $0.041(2)$ & $0.027(2)$ & $-0.001(2)$ & $-0.005(2)$ & $0.000(2)$ \\
\hline C19 & $0.040(3)$ & $0.042(2)$ & $0.035(2)$ & $-0.013(2)$ & $-0.004(2)$ & $0.001(2)$ \\
\hline $\mathrm{C} 20$ & $0.041(3)$ & $0.031(2)$ & $0.031(2)$ & $-0.007(2)$ & $0.002(2)$ & $0.008(2)$ \\
\hline $\mathrm{C} 21$ & $0.033(2)$ & $0.035(2)$ & $0.021(2)$ & $0.002(2)$ & $-0.005(2)$ & $0.006(2)$ \\
\hline $\mathrm{C} 22$ & $0.034(2)$ & $0.033(2)$ & $0.024(2)$ & $0.005(1)$ & $-0.001(2)$ & $0.001(2)$ \\
\hline $\mathrm{C} 23$ & $0.037(2)$ & $0.034(2)$ & $0.031(2)$ & $0.002(2)$ & $-0.005(2)$ & $0.006(2)$ \\
\hline $\mathrm{C} 24$ & $0.029(2)$ & $0.024(2)$ & $0.027(2)$ & $0.003(1)$ & $0.003(2)$ & $0.003(2)$ \\
\hline $\mathrm{C} 25$ & $0.023(2)$ & $0.029(2)$ & $0.024(2)$ & $0.001(1)$ & $0.004(2)$ & $0.004(1)$ \\
\hline
\end{tabular}




$\begin{array}{lllllll}\text { C26 } & 0.024(2) & 0.028(2) & 0.021(2) & 0.005(1) & 0.001(2) & -0.001(2) \\ \text { C27 } & 0.034(2) & 0.031(2) & 0.024(2) & 0.008(2) & 0.004(2) & 0.007(2) \\ \text { C28 } & 0.041(2) & 0.038(2) & 0.025(2) & -0.005(2) & -0.002(2) & -0.002(2) \\ \text { C29 } & 0.050(3) & 0.025(2) & 0.029(2) & 0.000(2) & 0.007(2) & 0.004(2) \\ \text { C30 } & 0.032(2) & 0.038(2) & 0.025(2) & 0.007(2) & 0.001(2) & 0.009(2) \\ \text { C31 } & 0.029(2) & 0.027(2) & 0.022(2) & 0.001(2) & 0.001(2) & 0.000(2) \\ \text { C32 } & 0.045(3) & 0.038(2) & 0.025(2) & 0.001(2) & -0.002(2) & 0.007(2) \\ \text { C33 } & 0.055(3) & 0.052(3) & 0.027(2) & 0.006(2) & -0.014(2) & -0.013(2) \\ \text { C34 } & 0.027(2) & 0.025(2) & 0.029(2) & 0.003(2) & -0.001(2) & 0.004(2) \\ \text { C35 } & 0.024(2) & 0.025(2) & 0.023(2) & 0.000(1) & -0.001(2) & -0.001(1) \\ \text { C36 } & 0.025(2) & 0.019(2) & 0.026(2) & 0.001(1) & 0.000(2) & 0.000(2) \\ \text { C37 } & 0.028(2) & 0.023(2) & 0.024(2) & 0.004(2) & 0.000(2) & 0.002(2) \\ \text { C38 } & 0.027(2) & 0.037(2) & 0.024(2) & -0.004(2) & -0.002(2) & -0.007(2) \\ \text { C39 } & 0.039(3) & 0.040(2) & 0.040(2) & -0.016(2) & -0.005(2) & 0.014(2) \\ \text { C40 } & 0.032(2) & 0.034(2) & 0.036(2) & -0.007(2) & -0.007(2) & 0.016(2)\end{array}$

The general temperature factor expression:

$\exp \left(-2 \Pi^{2}\left(a^{2} U_{11} h^{2}+b^{2} U_{22} k^{2}+c^{2} U_{33} l^{2}+2 a b U_{12} h k+2 a c U_{13} h l+2 b c U_{23} k l\right)\right)$ 
Table S-4. Bond Lengths ( $\AA$ )

\begin{tabular}{|c|c|c|c|c|c|}
\hline atom & atom & distance & atom & atom & distance \\
\hline $\mathrm{Br} 1$ & $\mathrm{C} 18$ & $1.888(3)$ & $\mathrm{Br} 2$ & C38 & $1.905(3)$ \\
\hline O1 & $\mathrm{C} 13$ & $1.406(3)$ & $\mathrm{O} 1$ & $\mathrm{C} 12$ & $1.421(4)$ \\
\hline $\mathrm{O} 2$ & N4 & $1.225(4)$ & $\mathrm{O} 3$ & N4 & $1.255(4)$ \\
\hline $\mathrm{O} 21$ & C33 & $1.417(4)$ & $\mathrm{O} 21$ & C32 & $1.418(4)$ \\
\hline $\mathrm{O} 22$ & N24 & $1.237(4)$ & $\mathrm{O} 23$ & N24 & $1.237(4)$ \\
\hline N1 & $\mathrm{C} 4$ & $1.357(5)$ & N1 & $\mathrm{C} 11$ & $1.387(5)$ \\
\hline N1 & $\mathrm{C} 1$ & $1.452(4)$ & $\mathrm{N} 2$ & $\mathrm{C} 14$ & $1.274(5)$ \\
\hline N2 & N3 & $1.383(4)$ & N3 & $\mathrm{C} 15$ & $1.345(4)$ \\
\hline N3 & H3 & $0.80(3)$ & N4 & C16 & $1.429(4)$ \\
\hline N21 & $\mathrm{C} 24$ & $1.364(4)$ & N21 & C31 & $1.375(4)$ \\
\hline N21 & $\mathrm{C} 21$ & $1.456(4)$ & N22 & C34 & $1.282(4)$ \\
\hline N22 & $\mathrm{N} 23$ & $1.387(4)$ & N23 & C35 & $1.365(4)$ \\
\hline N23 & $\mathrm{H} 23$ & $0.90(3)$ & N24 & $\mathrm{C} 36$ & $1.429(4)$ \\
\hline $\mathrm{C} 1$ & $\mathrm{C} 2$ & $1.557(5)$ & $\mathrm{C} 1$ & H1A & 0.9900 \\
\hline $\mathrm{C} 1$ & H1B & 0.9900 & $\mathrm{C} 2$ & $\mathrm{C} 12$ & $1.500(4)$ \\
\hline $\mathrm{C} 2$ & C3 & $1.543(4)$ & $\mathrm{C} 2$ & $\mathrm{H} 2 \mathrm{~A}$ & 1.0000 \\
\hline C3 & $\mathrm{C} 4$ & $1.502(5)$ & $\mathrm{C} 3$ & $\mathrm{H} 3 \mathrm{~A}$ & 0.9900 \\
\hline $\mathrm{C} 3$ & H3B & 0.9900 & $\mathrm{C} 4$ & $\mathrm{C} 5$ & $1.372(5)$ \\
\hline C5 & $\mathrm{C} 14$ & $1.429(5)$ & $\mathrm{C} 5$ & C6 & $1.454(5)$ \\
\hline C6 & C7 & $1.387(5)$ & C6 & C11 & $1.424(5)$ \\
\hline C7 & C8 & $1.364(6)$ & C7 & $\mathrm{H} 7 \mathrm{~A}$ & 0.9500 \\
\hline C8 & C9 & $1.418(6)$ & $\mathrm{C} 8$ & H8A & 0.9500 \\
\hline C9 & $\mathrm{C} 10$ & $1.378(5)$ & C9 & H9A & 0.9500 \\
\hline C10 & C11 & $1.367(5)$ & $\mathrm{C} 10$ & H10A & 0.9500 \\
\hline $\mathrm{C} 12$ & $\mathrm{H} 12 \mathrm{~A}$ & 0.9900 & $\mathrm{C} 12$ & H12B & 0.9900 \\
\hline $\mathrm{C} 13$ & H13A & 0.9800 & $\mathrm{C} 13$ & H13B & 0.9800 \\
\hline $\mathrm{C} 13$ & $\mathrm{H} 13 \mathrm{C}$ & 0.9800 & $\mathrm{C} 14$ & H14A & 0.9500 \\
\hline C15 & C16 & $1.403(5)$ & $\mathrm{C} 15$ & $\mathrm{C} 20$ & $1.416(5)$ \\
\hline C16 & C17 & $1.396(5)$ & $\mathrm{C} 17$ & $\mathrm{C} 18$ & $1.362(6)$ \\
\hline $\mathrm{C} 17$ & H17A & 0.9500 & $\mathrm{C} 18$ & C19 & $1.393(6)$ \\
\hline C19 & C20 & $1.352(5)$ & C19 & H19A & 0.9500 \\
\hline C20 & $\mathrm{H} 20 \mathrm{~A}$ & 0.9500 & $\mathrm{C} 21$ & $\mathrm{C} 22$ & $1.545(5)$ \\
\hline $\mathrm{C} 21$ & $\mathrm{H} 21 \mathrm{~A}$ & 0.9900 & $\mathrm{C} 21$ & $\mathrm{H} 21 \mathrm{~B}$ & 0.9900 \\
\hline $\mathrm{C} 22$ & C32 & $1.501(4)$ & $\mathrm{C} 22$ & $\mathrm{C} 23$ & $1.552(5)$ \\
\hline $\mathrm{C} 22$ & $\mathrm{H} 22 \mathrm{~A}$ & 1.0000 & $\mathrm{C} 23$ & C24 & $1.489(5)$ \\
\hline $\mathrm{C} 23$ & $\mathrm{H} 23 \mathrm{~A}$ & 0.9900 & $\mathrm{C} 23$ & $\mathrm{H} 23 \mathrm{~B}$ & 0.9900 \\
\hline $\mathrm{C} 24$ & C25 & $1.375(5)$ & $\mathrm{C} 25$ & $\mathrm{C} 26$ & $1.428(5)$ \\
\hline $\mathrm{C} 25$ & C34 & $1.444(5)$ & $\mathrm{C} 26$ & C31 & $1.398(5)$ \\
\hline $\mathrm{C} 26$ & C27 & $1.407(5)$ & $\mathrm{C} 27$ & $\mathrm{C} 28$ & $1.382(6)$ \\
\hline $\mathrm{C} 27$ & $\mathrm{H} 27 \mathrm{~A}$ & 0.9500 & $\mathrm{C} 28$ & $\mathrm{C} 29$ & $1.389(5)$ \\
\hline C28 & $\mathrm{H} 28 \mathrm{~A}$ & 0.9500 & $\mathrm{C} 29$ & C30 & $1.367(5)$ \\
\hline C29 & H29A & 0.9500 & $\mathrm{C} 30$ & C31 & $1.410(6)$ \\
\hline
\end{tabular}




$\begin{array}{llllll}\text { C30 } & \text { H30A } & 0.9500 & \text { C32 } & \text { H32A } & 0.9900 \\ \text { C32 } & \text { H32B } & 0.9900 & \text { C33 } & \text { H33A } & 0.9800 \\ \text { C33 } & \text { H33B } & 0.9800 & \text { C33 } & \text { H33C } & 0.9800 \\ \text { C34 } & \text { H34A } & 0.9500 & \text { C35 } & \text { C40 } & 1.404(5) \\ \text { C35 } & \text { C36 } & 1.419(5) & \text { C36 } & \text { C37 } & 1.392(5) \\ \text { C37 } & \text { C38 } & 1.359(5) & \text { C37 } & \text { H37A } & 0.9500 \\ \text { C38 } & \text { C39 } & 1.378(6) & \text { C39 } & \text { C40 } & 1.355(5) \\ \text { C39 } & \text { H39A } & 0.9500 & \text { C40 } & \text { H40A } & 0.9500\end{array}$


Table S-5. Bond Angles $\left({ }^{\circ}\right)$

\begin{tabular}{|c|c|c|c|c|c|c|c|}
\hline atom & atom & atom & angle & atom & atom & atom & angle \\
\hline $\mathrm{C} 13$ & $\mathrm{O} 1$ & $\mathrm{C} 12$ & $112.5(3)$ & C33 & $\mathrm{O} 21$ & C32 & $111.2(3)$ \\
\hline $\mathrm{C} 4$ & N1 & $\mathrm{C} 11$ & $110.0(3)$ & $\mathrm{C} 4$ & N1 & $\mathrm{C} 1$ & $115.2(3)$ \\
\hline C11 & N1 & $\mathrm{C} 1$ & $134.4(3)$ & C14 & N2 & N3 & $115.6(3)$ \\
\hline C15 & N3 & $\mathrm{N} 2$ & $119.3(3)$ & $\mathrm{C} 15$ & N3 & H3 & $113(3)$ \\
\hline N2 & N3 & $\mathrm{H} 3$ & $128(3)$ & $\mathrm{O} 2$ & N4 & $\mathrm{O} 3$ & $121.5(3)$ \\
\hline $\mathrm{O} 2$ & N4 & $\mathrm{C} 16$ & $120.4(3)$ & $\mathrm{O} 3$ & N4 & $\mathrm{C} 16$ & $118.1(3)$ \\
\hline C24 & $\mathrm{N} 21$ & C31 & $109.3(3)$ & $\mathrm{C} 24$ & $\mathrm{~N} 21$ & $\mathrm{C} 21$ & $114.5(3)$ \\
\hline C31 & $\mathrm{N} 21$ & $\mathrm{C} 21$ & $136.2(3)$ & C34 & $\mathrm{N} 22$ & $\mathrm{~N} 23$ & $114.7(3)$ \\
\hline C35 & $\mathrm{N} 23$ & N22 & $117.5(3)$ & C35 & $\mathrm{N} 23$ & $\mathrm{H} 23$ & $123(2)$ \\
\hline N22 & $\mathrm{N} 23$ & $\mathrm{H} 23$ & $118(2)$ & $\mathrm{O} 22$ & $\mathrm{~N} 24$ & $\mathrm{O} 23$ & $122.6(3)$ \\
\hline $\mathrm{O} 22$ & N24 & C36 & $118.9(3)$ & $\mathrm{O} 23$ & $\mathrm{~N} 24$ & C36 & $118.5(3)$ \\
\hline N1 & $\mathrm{C} 1$ & $\mathrm{C} 2$ & $101.5(3)$ & N1 & $\mathrm{C} 1$ & $\mathrm{H} 1 \mathrm{~A}$ & 111.5 \\
\hline $\mathrm{C} 2$ & $\mathrm{C} 1$ & H1A & 111.5 & N1 & $\mathrm{C} 1$ & H1B & 111.5 \\
\hline $\mathrm{C} 2$ & $\mathrm{C} 1$ & H1B & 111.5 & $\mathrm{H} 1 \mathrm{~A}$ & $\mathrm{C} 1$ & H1B & 109.3 \\
\hline $\mathrm{C} 12$ & $\mathrm{C} 2$ & $\mathrm{C} 3$ & $111.5(3)$ & $\mathrm{C} 12$ & $\mathrm{C} 2$ & $\mathrm{C} 1$ & $112.7(3)$ \\
\hline C3 & $\mathrm{C} 2$ & $\mathrm{C} 1$ & $105.5(3)$ & $\mathrm{C} 12$ & $\mathrm{C} 2$ & $\mathrm{H} 2 \mathrm{~A}$ & 109.0 \\
\hline C3 & $\mathrm{C} 2$ & $\mathrm{H} 2 \mathrm{~A}$ & 109.0 & $\mathrm{C} 1$ & $\mathrm{C} 2$ & $\mathrm{H} 2 \mathrm{~A}$ & 109.0 \\
\hline $\mathrm{C} 4$ & $\mathrm{C} 3$ & $\mathrm{C} 2$ & $103.5(3)$ & $\mathrm{C} 4$ & $\mathrm{C} 3$ & $\mathrm{H} 3 \mathrm{~A}$ & 111.1 \\
\hline $\mathrm{C} 2$ & $\mathrm{C} 3$ & $\mathrm{H} 3 \mathrm{~A}$ & 111.1 & $\mathrm{C} 4$ & $\mathrm{C} 3$ & $\mathrm{H} 3 \mathrm{~B}$ & 111.1 \\
\hline $\mathrm{C} 2$ & $\mathrm{C} 3$ & H3B & 111.1 & $\mathrm{H} 3 \mathrm{~A}$ & C3 & $\mathrm{H} 3 \mathrm{~B}$ & 109.0 \\
\hline N1 & $\mathrm{C} 4$ & C5 & $110.9(3)$ & N1 & $\mathrm{C} 4$ & C3 & $109.0(3)$ \\
\hline C5 & $\mathrm{C} 4$ & $\mathrm{C} 3$ & $140.0(4)$ & $\mathrm{C} 4$ & C5 & C14 & $126.3(4)$ \\
\hline $\mathrm{C} 4$ & $\mathrm{C} 5$ & C6 & $105.4(3)$ & $\mathrm{C} 14$ & C5 & C6 & $128.1(3)$ \\
\hline C7 & C6 & $\mathrm{C} 11$ & $117.7(3)$ & $\mathrm{C} 7$ & C6 & C5 & $134.8(3)$ \\
\hline C11 & C6 & C5 & $107.4(3)$ & C8 & C7 & C6 & $120.3(4)$ \\
\hline $\mathrm{C} 8$ & $\mathrm{C} 7$ & H7A & 119.8 & C6 & $\mathrm{C} 7$ & $\mathrm{H} 7 \mathrm{~A}$ & 119.8 \\
\hline C7 & $\mathrm{C} 8$ & C9 & $120.7(4)$ & C7 & $\mathrm{C} 8$ & H8A & 119.6 \\
\hline C9 & $\mathrm{C} 8$ & H8A & 119.6 & $\mathrm{C} 10$ & C9 & C8 & $120.3(4)$ \\
\hline C10 & C9 & H9A & 119.9 & C8 & C9 & H9A & 119.9 \\
\hline C11 & $\mathrm{C} 10$ & C9 & $118.2(4)$ & $\mathrm{C} 11$ & C10 & H10A & 120.9 \\
\hline C9 & $\mathrm{C} 10$ & $\mathrm{H} 10 \mathrm{~A}$ & 120.9 & $\mathrm{C} 10$ & $\mathrm{C} 11$ & N1 & $131.0(4)$ \\
\hline $\mathrm{C} 10$ & $\mathrm{C} 11$ & C6 & $122.7(4)$ & N1 & $\mathrm{C} 11$ & C6 & $106.2(3)$ \\
\hline $\mathrm{O} 1$ & $\mathrm{C} 12$ & $\mathrm{C} 2$ & $115.1(3)$ & $\mathrm{O} 1$ & $\mathrm{C} 12$ & H12A & 108.5 \\
\hline $\mathrm{C} 2$ & $\mathrm{C} 12$ & $\mathrm{H} 12 \mathrm{~A}$ & 108.5 & $\mathrm{O} 1$ & $\mathrm{C} 12$ & H12B & 108.5 \\
\hline $\mathrm{C} 2$ & $\mathrm{C} 12$ & H12B & 108.5 & $\mathrm{H} 12 \mathrm{~A}$ & $\mathrm{C} 12$ & H12B & 107.5 \\
\hline $\mathrm{O} 1$ & $\mathrm{C} 13$ & $\mathrm{H} 13 \mathrm{~A}$ & 109.5 & $\mathrm{O} 1$ & $\mathrm{C} 13$ & H13B & 109.5 \\
\hline H13A & $\mathrm{C} 13$ & H13B & 109.5 & O1 & $\mathrm{C} 13$ & $\mathrm{H} 13 \mathrm{C}$ & 109.5 \\
\hline H13A & $\mathrm{C} 13$ & $\mathrm{H} 13 \mathrm{C}$ & 109.5 & H13B & C13 & $\mathrm{H} 13 \mathrm{C}$ & 109.5 \\
\hline N2 & C14 & $\mathrm{C} 5$ & $121.5(3)$ & N2 & $\mathrm{C} 14$ & H14A & 119.2 \\
\hline C5 & C14 & H14A & 119.2 & N3 & C15 & C16 & $124.9(3)$ \\
\hline N3 & $\mathrm{C} 15$ & $\mathrm{C} 20$ & $119.9(3)$ & $\mathrm{C} 16$ & C15 & $\mathrm{C} 20$ & $115.2(3)$ \\
\hline $\mathrm{C} 17$ & $\mathrm{C} 16$ & C15 & $122.9(3)$ & $\mathrm{C} 17$ & $\mathrm{C} 16$ & N4 & $114.8(3)$ \\
\hline
\end{tabular}




\begin{tabular}{|c|c|c|c|c|c|c|c|}
\hline $\mathrm{C} 15$ & $\mathrm{C} 16$ & N4 & $122.2(3)$ & $\mathrm{C} 18$ & $\mathrm{C} 17$ & C16 & $119.3(3)$ \\
\hline C18 & $\mathrm{C} 17$ & H17A & 120.4 & $\mathrm{C} 16$ & $\mathrm{C} 17$ & H17A & 120.4 \\
\hline $\mathrm{C} 17$ & $\mathrm{C} 18$ & C19 & $119.2(4)$ & $\mathrm{C} 17$ & $\mathrm{C} 18$ & $\mathrm{Br} 1$ & $120.5(3)$ \\
\hline C19 & C18 & $\mathrm{Br} 1$ & $120.3(3)$ & $\mathrm{C} 20$ & C19 & C18 & $121.7(4)$ \\
\hline $\mathrm{C} 20$ & C19 & H19A & 119.2 & $\mathrm{C} 18$ & C19 & H19A & 119.2 \\
\hline C19 & $\mathrm{C} 20$ & C15 & $121.6(4)$ & C19 & $\mathrm{C} 20$ & $\mathrm{H} 20 \mathrm{~A}$ & 119.2 \\
\hline $\mathrm{C} 15$ & $\mathrm{C} 20$ & $\mathrm{H} 20 \mathrm{~A}$ & 119.2 & N21 & $\mathrm{C} 21$ & $\mathrm{C} 22$ & $103.0(3)$ \\
\hline N21 & $\mathrm{C} 21$ & $\mathrm{H} 21 \mathrm{~A}$ & 111.2 & $\mathrm{C} 22$ & $\mathrm{C} 21$ & $\mathrm{H} 21 \mathrm{~A}$ & 111.2 \\
\hline N21 & $\mathrm{C} 21$ & $\mathrm{H} 21 \mathrm{~B}$ & 111.2 & $\mathrm{C} 22$ & $\mathrm{C} 21$ & $\mathrm{H} 21 \mathrm{~B}$ & 111.2 \\
\hline $\mathrm{H} 21 \mathrm{~A}$ & $\mathrm{C} 21$ & $\mathrm{H} 21 \mathrm{~B}$ & 109.1 & $\mathrm{C} 32$ & $\mathrm{C} 22$ & $\mathrm{C} 21$ & $113.9(3)$ \\
\hline C32 & $\mathrm{C} 22$ & $\mathrm{C} 23$ & $111.5(3)$ & $\mathrm{C} 21$ & $\mathrm{C} 22$ & $\mathrm{C} 23$ & $106.5(3)$ \\
\hline C32 & $\mathrm{C} 22$ & $\mathrm{H} 22 \mathrm{~A}$ & 108.3 & $\mathrm{C} 21$ & $\mathrm{C} 22$ & $\mathrm{H} 22 \mathrm{~A}$ & 108.3 \\
\hline $\mathrm{C} 23$ & $\mathrm{C} 22$ & $\mathrm{H} 22 \mathrm{~A}$ & 108.3 & $\mathrm{C} 24$ & $\mathrm{C} 23$ & $\mathrm{C} 22$ & $103.8(3)$ \\
\hline $\mathrm{C} 24$ & $\mathrm{C} 23$ & $\mathrm{H} 23 \mathrm{~A}$ & 111.0 & $\mathrm{C} 22$ & $\mathrm{C} 23$ & $\mathrm{H} 23 \mathrm{~A}$ & 111.0 \\
\hline C24 & $\mathrm{C} 23$ & $\mathrm{H} 23 \mathrm{~B}$ & 111.0 & $\mathrm{C} 22$ & $\mathrm{C} 23$ & $\mathrm{H} 23 \mathrm{~B}$ & 111.0 \\
\hline $\mathrm{H} 23 \mathrm{~A}$ & $\mathrm{C} 23$ & $\mathrm{H} 23 \mathrm{~B}$ & 109.0 & N21 & $\mathrm{C} 24$ & $\mathrm{C} 25$ & $109.8(3)$ \\
\hline N21 & $\mathrm{C} 24$ & $\mathrm{C} 23$ & $110.1(3)$ & $\mathrm{C} 25$ & $\mathrm{C} 24$ & $\mathrm{C} 23$ & $140.1(3)$ \\
\hline $\mathrm{C} 24$ & $\mathrm{C} 25$ & $\mathrm{C} 26$ & $105.9(3)$ & $\mathrm{C} 24$ & $\mathrm{C} 25$ & C34 & $124.6(3)$ \\
\hline $\mathrm{C} 26$ & $\mathrm{C} 25$ & C34 & $129.5(3)$ & C31 & $\mathrm{C} 26$ & $\mathrm{C} 27$ & 118.1(3) \\
\hline C31 & $\mathrm{C} 26$ & $\mathrm{C} 25$ & $107.8(3)$ & $\mathrm{C} 27$ & $\mathrm{C} 26$ & $\mathrm{C} 25$ & $134.1(3)$ \\
\hline $\mathrm{C} 28$ & $\mathrm{C} 27$ & $\mathrm{C} 26$ & $118.7(4)$ & $\mathrm{C} 28$ & $\mathrm{C} 27$ & $\mathrm{H} 27 \mathrm{~A}$ & 120.7 \\
\hline C26 & $\mathrm{C} 27$ & $\mathrm{H} 27 \mathrm{~A}$ & 120.7 & $\mathrm{C} 27$ & $\mathrm{C} 28$ & C29 & $121.5(4)$ \\
\hline $\mathrm{C} 27$ & $\mathrm{C} 28$ & $\mathrm{H} 28 \mathrm{~A}$ & 119.2 & $\mathrm{C} 29$ & $\mathrm{C} 28$ & H28A & 119.2 \\
\hline C30 & $\mathrm{C} 29$ & $\mathrm{C} 28$ & $122.0(4)$ & C30 & $\mathrm{C} 29$ & H29A & 119.0 \\
\hline C28 & C29 & H29A & 119.0 & $\mathrm{C} 29$ & $\mathrm{C} 30$ & C31 & $116.3(4)$ \\
\hline C29 & $\mathrm{C} 30$ & H30A & 121.9 & C31 & $\mathrm{C} 30$ & H30A & 121.9 \\
\hline N21 & C31 & $\mathrm{C} 26$ & $107.1(3)$ & N21 & C31 & C30 & $129.5(3)$ \\
\hline $\mathrm{C} 26$ & C31 & C30 & $123.4(3)$ & $\mathrm{O} 21$ & C32 & $\mathrm{C} 22$ & $109.3(3)$ \\
\hline $\mathrm{O} 21$ & C32 & H32A & 109.8 & $\mathrm{C} 22$ & C32 & H32A & 109.8 \\
\hline $\mathrm{O} 21$ & C32 & H32B & 109.8 & $\mathrm{C} 22$ & C32 & H32B & 109.8 \\
\hline H32A & C32 & H32B & 108.3 & $\mathrm{O} 21$ & $\mathrm{C} 33$ & H33A & 109.5 \\
\hline $\mathrm{O} 21$ & C33 & H33B & 109.5 & H33A & $\mathrm{C} 33$ & H33B & 109.5 \\
\hline $\mathrm{O} 21$ & $\mathrm{C} 33$ & H33C & 109.5 & H33A & C33 & H33C & 109.5 \\
\hline H33B & C33 & $\mathrm{H} 33 \mathrm{C}$ & 109.5 & N22 & C34 & $\mathrm{C} 25$ & $119.9(3)$ \\
\hline N22 & C34 & H34A & 120.1 & $\mathrm{C} 25$ & C34 & H34A & 120.1 \\
\hline $\mathrm{N} 23$ & $\mathrm{C} 35$ & $\mathrm{C} 40$ & 119.1(3) & N23 & $\mathrm{C} 35$ & C36 & $124.8(3)$ \\
\hline $\mathrm{C} 40$ & $\mathrm{C} 35$ & $\mathrm{C} 36$ & $116.0(3)$ & C37 & $\mathrm{C} 36$ & $\mathrm{C} 35$ & $121.2(3)$ \\
\hline C37 & $\mathrm{C} 36$ & $\mathrm{~N} 24$ & $117.1(3)$ & C35 & $\mathrm{C} 36$ & N24 & $121.7(3)$ \\
\hline C38 & C37 & C36 & $119.4(3)$ & C38 & C37 & H37A & 120.3 \\
\hline C36 & C37 & H37A & 120.3 & C37 & C38 & C39 & $120.9(3)$ \\
\hline C37 & C38 & $\mathrm{Br} 2$ & $120.0(3)$ & C39 & C38 & $\mathrm{Br} 2$ & 119.1(3) \\
\hline $\mathrm{C} 40$ & C39 & C38 & $120.3(4)$ & $\mathrm{C} 40$ & C39 & H39A & 119.9 \\
\hline C38 & C39 & H39A & 119.9 & C39 & $\mathrm{C} 40$ & C35 & $122.1(4)$ \\
\hline C39 & $\mathrm{C} 40$ & $\mathrm{H} 40 \mathrm{~A}$ & 119.0 & $\mathrm{C} 35$ & $\mathrm{C} 40$ & $\mathrm{H} 40 \mathrm{~A}$ & 119.0 \\
\hline
\end{tabular}


Table S-6. Torsion Angles ( $\left.{ }^{\circ}\right)$

atom atom atom atom angle atom atom atom atom angle

\begin{tabular}{|c|c|c|c|c|c|c|c|c|c|}
\hline C14 & N2 & N3 & C15 & $-172.7(3)$ & C34 & $\mathrm{N} 22$ & $\mathrm{~N} 23$ & C35 & 172.7(3) \\
\hline $\mathrm{C} 4$ & N1 & $\mathrm{C} 1$ & $\mathrm{C} 2$ & $16.2(4)$ & $\mathrm{C} 11$ & N1 & $\mathrm{C} 1$ & $\mathrm{C} 2$ & $-172.8(3)$ \\
\hline N1 & $\mathrm{C} 1$ & $\mathrm{C} 2$ & $\mathrm{C} 12$ & $-144.1(3)$ & N1 & $\mathrm{C} 1$ & $\mathrm{C} 2$ & $\mathrm{C} 3$ & $-22.3(3)$ \\
\hline $\mathrm{C} 12$ & $\mathrm{C} 2$ & $\mathrm{C} 3$ & $\mathrm{C} 4$ & $143.7(3)$ & $\mathrm{C} 1$ & $\mathrm{C} 2$ & $\mathrm{C} 3$ & $\mathrm{C} 4$ & $21.1(3)$ \\
\hline $\mathrm{C} 11$ & N1 & $\mathrm{C} 4$ & $\mathrm{C} 5$ & $1.9(4)$ & $\mathrm{C} 1$ & N1 & $\mathrm{C} 4$ & $\mathrm{C} 5$ & 175.1(3) \\
\hline $\mathrm{C} 11$ & N1 & $\mathrm{C} 4$ & $\mathrm{C} 3$ & $-176.2(3)$ & $\mathrm{C} 1$ & N1 & $\mathrm{C} 4$ & $\mathrm{C} 3$ & $-3.0(4)$ \\
\hline C2 & C3 & $\mathrm{C} 4$ & N1 & $-11.9(4)$ & $\mathrm{C} 2$ & $\mathrm{C} 3$ & $\mathrm{C} 4$ & $\mathrm{C} 5$ & $170.9(4)$ \\
\hline N1 & $\mathrm{C} 4$ & $\mathrm{C} 5$ & C14 & $-176.1(3)$ & $\mathrm{C} 3$ & $\mathrm{C} 4$ & $\mathrm{C} 5$ & C14 & $1.1(7)$ \\
\hline N1 & $\mathrm{C} 4$ & $\mathrm{C} 5$ & C6 & $-0.2(4)$ & $\mathrm{C} 3$ & $\mathrm{C} 4$ & $\mathrm{C} 5$ & C6 & $176.9(4)$ \\
\hline C4 & C5 & C6 & C7 & $176.4(4)$ & C14 & $\mathrm{C} 5$ & C6 & C7 & $-7.9(6)$ \\
\hline $\mathrm{C} 4$ & $\mathrm{C} 5$ & C6 & C11 & $-1.5(4)$ & $\mathrm{C} 14$ & $\mathrm{C} 5$ & C6 & C11 & 174.3(3) \\
\hline $\mathrm{C} 11$ & C6 & $\mathrm{C} 7$ & $\mathrm{C} 8$ & $-1.0(5)$ & $\mathrm{C} 5$ & C6 & $\mathrm{C} 7$ & $\mathrm{C} 8$ & $-178.7(4)$ \\
\hline C6 & C7 & $\mathrm{C} 8$ & C9 & $-0.6(5)$ & C7 & $\mathrm{C} 8$ & C9 & $\mathrm{C} 10$ & $0.9(6)$ \\
\hline $\mathrm{C} 8$ & C9 & $\mathrm{C} 10$ & $\mathrm{C} 11$ & $0.4(6)$ & C9 & $\mathrm{C} 10$ & C11 & N1 & $175.5(4)$ \\
\hline C9 & $\mathrm{C} 10$ & $\mathrm{C} 11$ & C6 & $-2.1(5)$ & $\mathrm{C} 4$ & N1 & $\mathrm{C} 11$ & $\mathrm{C} 10$ & 179.3(3) \\
\hline $\mathrm{C} 1$ & N1 & $\mathrm{C} 11$ & $\mathrm{C} 10$ & $8.0(6)$ & $\mathrm{C} 4$ & N1 & C11 & C6 & $-2.7(4)$ \\
\hline $\mathrm{C} 1$ & N1 & $\mathrm{C} 11$ & C6 & $-174.1(3)$ & $\mathrm{C} 7$ & C6 & $\mathrm{C} 11$ & $\mathrm{C} 10$ & $2.4(5)$ \\
\hline C5 & C6 & $\mathrm{C} 11$ & $\mathrm{C} 10$ & $-179.3(3)$ & C7 & C6 & $\mathrm{C} 11$ & N1 & $-175.7(3)$ \\
\hline C5 & C6 & C11 & N1 & $2.6(3)$ & $\mathrm{C} 13$ & O1 & $\mathrm{C} 12$ & $\mathrm{C} 2$ & $-81.8(4)$ \\
\hline C3 & $\mathrm{C} 2$ & $\mathrm{C} 12$ & $\mathrm{O} 1$ & $-176.1(2)$ & $\mathrm{C} 1$ & $\mathrm{C} 2$ & $\mathrm{C} 12$ & O1 & $-57.7(3)$ \\
\hline N3 & $\mathrm{N} 2$ & C14 & $\mathrm{C} 5$ & $179.6(3)$ & $\mathrm{C} 4$ & $\mathrm{C} 5$ & C14 & $\mathrm{N} 2$ & $170.4(3)$ \\
\hline C6 & $\mathrm{C} 5$ & C14 & N2 & $-4.5(5)$ & $\mathrm{N} 2$ & N3 & $\mathrm{C} 15$ & $\mathrm{C} 16$ & $170.8(3)$ \\
\hline N2 & N3 & $\mathrm{C} 15$ & $\mathrm{C} 20$ & $-8.1(5)$ & N3 & C15 & $\mathrm{C} 16$ & $\mathrm{C} 17$ & $-178.5(3)$ \\
\hline $\mathrm{C} 20$ & $\mathrm{C} 15$ & $\mathrm{C} 16$ & $\mathrm{C} 17$ & $0.5(5)$ & N3 & C15 & $\mathrm{C} 16$ & N4 & $-1.2(5)$ \\
\hline $\mathrm{C} 20$ & $\mathrm{C} 15$ & $\mathrm{C} 16$ & N4 & $177.7(3)$ & $\mathrm{O} 2$ & N4 & $\mathrm{C} 16$ & $\mathrm{C} 17$ & $10.8(5)$ \\
\hline $\mathrm{O} 3$ & N4 & $\mathrm{C} 16$ & $\mathrm{C} 17$ & $-169.5(3)$ & $\mathrm{O} 2$ & N4 & $\mathrm{C} 16$ & $\mathrm{C} 15$ & $-166.7(3)$ \\
\hline $\mathrm{O} 3$ & N4 & $\mathrm{C} 16$ & $\mathrm{C} 15$ & $13.0(5)$ & $\mathrm{C} 15$ & $\mathrm{C} 16$ & $\mathrm{C} 17$ & $\mathrm{C} 18$ & $-3.3(5)$ \\
\hline N4 & $\mathrm{C} 16$ & $\mathrm{C} 17$ & $\mathrm{C} 18$ & $179.3(3)$ & $\mathrm{C} 16$ & $\mathrm{C} 17$ & $\mathrm{C} 18$ & $\mathrm{C} 19$ & $3.7(5)$ \\
\hline $\mathrm{C} 16$ & $\mathrm{C} 17$ & $\mathrm{C} 18$ & $\mathrm{Br} 1$ & $-176.9(3)$ & $\mathrm{C} 17$ & $\mathrm{C} 18$ & C19 & $\mathrm{C} 20$ & $-1.4(6)$ \\
\hline $\mathrm{Br} 1$ & $\mathrm{C} 18$ & C19 & $\mathrm{C} 20$ & $179.2(3)$ & $\mathrm{C} 18$ & C19 & $\mathrm{C} 20$ & $\mathrm{C} 15$ & $-1.5(6)$ \\
\hline N3 & $\mathrm{C} 15$ & $\mathrm{C} 20$ & C19 & $-179.1(4)$ & $\mathrm{C} 16$ & $\mathrm{C} 15$ & $\mathrm{C} 20$ & C19 & $1.9(5)$ \\
\hline $\mathrm{C} 24$ & N21 & $\mathrm{C} 21$ & $\mathrm{C} 22$ & $8.1(4)$ & C31 & N21 & $\mathrm{C} 21$ & $\mathrm{C} 22$ & $-172.9(3)$ \\
\hline N21 & $\mathrm{C} 21$ & $\mathrm{C} 22$ & C32 & $-136.5(3)$ & $\mathrm{N} 21$ & $\mathrm{C} 21$ & $\mathrm{C} 22$ & $\mathrm{C} 23$ & $-13.2(3)$ \\
\hline C32 & $\mathrm{C} 22$ & $\mathrm{C} 23$ & $\mathrm{C} 24$ & $138.5(3)$ & $\mathrm{C} 21$ & $\mathrm{C} 22$ & $\mathrm{C} 23$ & $\mathrm{C} 24$ & $13.7(3)$ \\
\hline $\mathrm{C} 31$ & N21 & $\mathrm{C} 24$ & $\mathrm{C} 25$ & $1.0(4)$ & $\mathrm{C} 21$ & $\mathrm{~N} 21$ & $\mathrm{C} 24$ & $\mathrm{C} 25$ & $-179.8(3)$ \\
\hline C31 & N21 & $\mathrm{C} 24$ & $\mathrm{C} 23$ & $-178.6(3)$ & $\mathrm{C} 21$ & $\mathrm{~N} 21$ & $\mathrm{C} 24$ & $\mathrm{C} 23$ & $0.6(4)$ \\
\hline $\mathrm{C} 22$ & $\mathrm{C} 23$ & $\mathrm{C} 24$ & N21 & $-9.1(4)$ & $\mathrm{C} 22$ & $\mathrm{C} 23$ & $\mathrm{C} 24$ & $\mathrm{C} 25$ & $171.5(4)$ \\
\hline N21 & $\mathrm{C} 24$ & $\mathrm{C} 25$ & $\mathrm{C} 26$ & $-1.6(4)$ & $\mathrm{C} 23$ & $\mathrm{C} 24$ & $\mathrm{C} 25$ & $\mathrm{C} 26$ & $177.8(4)$ \\
\hline N21 & $\mathrm{C} 24$ & $\mathrm{C} 25$ & C34 & $176.5(3)$ & $\mathrm{C} 23$ & $\mathrm{C} 24$ & $\mathrm{C} 25$ & C34 & $-4.2(7)$ \\
\hline $\mathrm{C} 24$ & $\mathrm{C} 25$ & $\mathrm{C} 26$ & C31 & $1.6(4)$ & C34 & $\mathrm{C} 25$ & $\mathrm{C} 26$ & $\mathrm{C} 31$ & $-176.3(3)$ \\
\hline $\mathrm{C} 24$ & $\mathrm{C} 25$ & $\mathrm{C} 26$ & $\mathrm{C} 27$ & $-175.8(4)$ & C34 & $\mathrm{C} 25$ & $\mathrm{C} 26$ & $\mathrm{C} 27$ & $6.3(6)$ \\
\hline $\mathrm{C} 31$ & $\mathrm{C} 26$ & $\mathrm{C} 27$ & $\mathrm{C} 28$ & $0.2(5)$ & $\mathrm{C} 25$ & $\mathrm{C} 26$ & $\mathrm{C} 27$ & $\mathrm{C} 28$ & $177.5(3)$ \\
\hline
\end{tabular}




$\begin{array}{llllllllll}\mathrm{C} 26 & \mathrm{C} 27 & \mathrm{C} 28 & \mathrm{C} 29 & 2.3(5) & \mathrm{C} 27 & \mathrm{C} 28 & \mathrm{C} 29 & \mathrm{C} 30 & -3.2(6) \\ \mathrm{C} 28 & \mathrm{C} 29 & \mathrm{C} 30 & \mathrm{C} 31 & 1.3(5) & \mathrm{C} 24 & \mathrm{~N} 21 & \mathrm{C} 31 & \mathrm{C} 26 & 0.1(4) \\ \mathrm{C} 21 & \mathrm{~N} 21 & \mathrm{C} 31 & \mathrm{C} 26 & -178.9(3) & \mathrm{C} 24 & \mathrm{~N} 21 & \mathrm{C} 31 & \mathrm{C} 30 & 178.9(3) \\ \mathrm{C} 21 & \mathrm{~N} 21 & \mathrm{C} 31 & \mathrm{C} 30 & -0.1(6) & \mathrm{C} 27 & \mathrm{C} 26 & \mathrm{C} 31 & \mathrm{~N} 21 & 176.8(3) \\ \mathrm{C} 25 & \mathrm{C} 26 & \mathrm{C} 31 & \mathrm{~N} 21 & -1.1(4) & \mathrm{C} 27 & \mathrm{C} 26 & \mathrm{C} 31 & \mathrm{C} 30 & -2.1(5) \\ \mathrm{C} 25 & \mathrm{C} 26 & \mathrm{C} 31 & \mathrm{C} 30 & -180.0(3) & \mathrm{C} 29 & \mathrm{C} 30 & \mathrm{C} 31 & \mathrm{~N} 21 & -177.4(3) \\ \mathrm{C} 29 & \mathrm{C} 30 & \mathrm{C} 31 & \mathrm{C} 26 & 1.3(5) & \mathrm{C} 33 & \mathrm{O} 21 & \mathrm{C} 32 & \mathrm{C} 22 & -178.0(3) \\ \mathrm{C} 21 & \mathrm{C} 22 & \mathrm{C} 32 & \mathrm{O} 21 & -59.0(4) & \mathrm{C} 23 & \mathrm{C} 22 & \mathrm{C} 32 & \mathrm{O} 21 & -179.6(3) \\ \mathrm{N} 23 & \mathrm{~N} 22 & \mathrm{C} 34 & \mathrm{C} 25 & -179.4(3) & \mathrm{C} 24 & \mathrm{C} 25 & \mathrm{C} 34 & \mathrm{~N} 22 & -170.2(3) \\ \mathrm{C} 26 & \mathrm{C} 25 & \mathrm{C} 34 & \mathrm{~N} 22 & 7.4(5) & \mathrm{N} 22 & \mathrm{~N} 23 & \mathrm{C} 35 & \mathrm{C} 40 & 13.1(5) \\ \mathrm{N} 22 & \mathrm{~N} 23 & \mathrm{C} 35 & \mathrm{C} 36 & -166.3(3) & \mathrm{N} 23 & \mathrm{C} 35 & \mathrm{C} 36 & \mathrm{C} 37 & 176.6(3) \\ \mathrm{C} 40 & \mathrm{C} 35 & \mathrm{C} 36 & \mathrm{C} 37 & -2.8(5) & \mathrm{N} 23 & \mathrm{C} 35 & \mathrm{C} 36 & \mathrm{~N} 24 & -1.4(5) \\ \mathrm{C} 40 & \mathrm{C} 35 & \mathrm{C} 36 & \mathrm{~N} 24 & 179.3(3) & \mathrm{O} 22 & \mathrm{~N} 24 & \mathrm{C} 36 & \mathrm{C} 37 & -11.5(5) \\ \mathrm{O} 23 & \mathrm{~N} 24 & \mathrm{C} 36 & \mathrm{C} 37 & 167.1(3) & \mathrm{O} 22 & \mathrm{~N} 24 & \mathrm{C} 36 & \mathrm{C} 35 & 166.5(3) \\ \mathrm{O} 23 & \mathrm{~N} 24 & \mathrm{C} 36 & \mathrm{C} 35 & -14.9(5) & \mathrm{C} 35 & \mathrm{C} 36 & \mathrm{C} 37 & \mathrm{C} 38 & 4.1(5) \\ \mathrm{N} 24 & \mathrm{C} 36 & \mathrm{C} 37 & \mathrm{C} 38 & -177.9(3) & \mathrm{C} 36 & \mathrm{C} 37 & \mathrm{C} 38 & \mathrm{C} 39 & -2.0(5) \\ \mathrm{C} 36 & \mathrm{C} 37 & \mathrm{C} 38 & \mathrm{Br} & 176.4(3) & \mathrm{C} 37 & \mathrm{C} 38 & \mathrm{C} 39 & \mathrm{C} 40 & -1.4(6) \\ \mathrm{Br} 2 & \mathrm{C} 38 & \mathrm{C} 39 & \mathrm{C} 40 & -179.8(3) & \mathrm{C} 38 & \mathrm{C} 39 & \mathrm{C} 40 & \mathrm{C} 35 & 2.7(6) \\ \mathrm{N} 23 & \mathrm{C} 35 & \mathrm{C} 40 & \mathrm{C} 39 & 180.0(4) & \mathrm{C} 36 & \mathrm{C} 35 & \mathrm{C} 40 & \mathrm{C} 39 & -0.6(5)\end{array}$

Table S-7. Hydrogen Bonds

Donor Hydrogen Acceptor D(D-H) $(\AA)$ D(H...A) $(\AA)$ D(D...A) $(\AA) \quad$ Angle (D-H...A) $\left({ }^{\circ}\right)$

$\begin{array}{lllllll}\mathrm{N}(3) & \mathrm{H}(3) & \mathrm{O}(3) & 0.80(3) & 1.98(4) & 2.628(4) & 137(3) \\ \mathrm{N}(3) & \mathrm{H}(3) & \mathrm{O}(23) & 0.80(3) & 2.65(3) & 3.373(4) & 152(3) \\ \mathrm{N}(23) & \mathrm{H}(23) & \mathrm{O}(3) & 0.90(3) & 2.45(3) & 3.322(4) & 166(3) \\ \mathrm{N}(23) & \mathrm{H}(23) & \mathrm{O}(23) & 0.90(3) & 2.08(4) & 2.648(4) & 120(3)\end{array}$

${ }^{1}$ van der Ent, A.; Onderdelinden, A. L. Inorg. Syn. 1973, 14, 93.

${ }^{2}$ Arnold, L. A.; Imbos, R.; Mandoli, A.; de Vries, A. H. M.; Naasz, R.; Feringa, B. L. Tetrahedron 2000, 56, 28652878.

${ }^{3}$ Adams, D. R. et al. Pyrroloindoles, Pyridoindoles, and Azopinoindoles as 5-HT $2 \mathrm{C}$ Agonists. US Patent 6,433,175 B1, August 13, 2002.

${ }^{4}$ Meyer, M. D.; Kruse, L. I. J. Org. Chem. 1984, 49, 3195-3199.

${ }^{5}$ Wang, J.; Rosingana, M.; Watson, D. J.; Dowdy, E. D.; Discordia, R. P.; Soundarajan, N.; Li, W.-S. Tetrahedron Lett. 2001, 42, 8935-8937.

${ }^{6}$ Wolfe, J. P.; Buchwald, S. L. J. Org. Chem. 2000, 65, 1144-1157. 

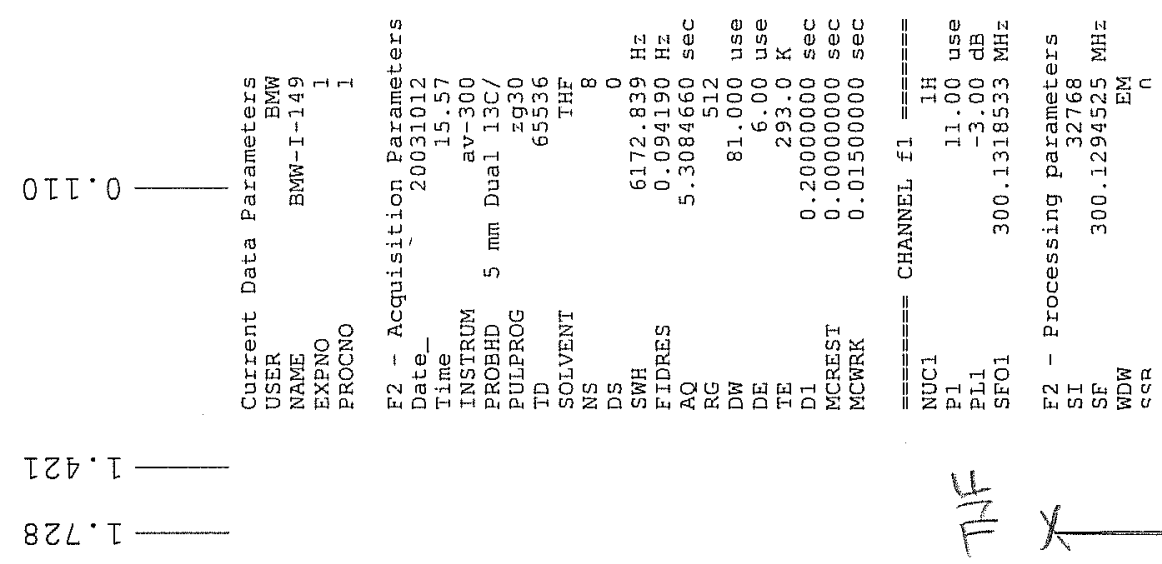

$8 \mathrm{~L}^{\circ} \mathrm{T}$

$\varepsilon \nabla 0^{\circ} Z$

$267 \cdot 2$

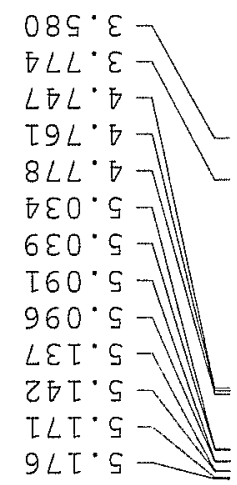

\section{$\angle 56^{\circ} S$}

$9 \angle 6^{\circ}$

$266^{\circ}$

OTO

रह० 9

$600 \cdot 9$

$990^{\circ} 9$

780.9

$66 \mathrm{~L} \cdot 9$

108.9

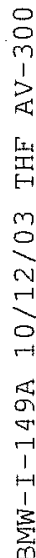

$628 \cdot 9$

$\angle \varepsilon 8 \cdot 9$

Zह[' $L$

$\angle S I^{\circ} L$

$28 T^{\circ} L$

$9 \tau Z \cdot L$

$5 \sigma Z^{\circ} L$

$99 Z^{\circ} L$

$062^{\circ} L$

$99 \varepsilon^{\circ} L$

$06 \varepsilon^{\circ} L L I \|$

$\left[L \sigma^{\circ} L\right]$

$6 L \sigma^{\circ} L$

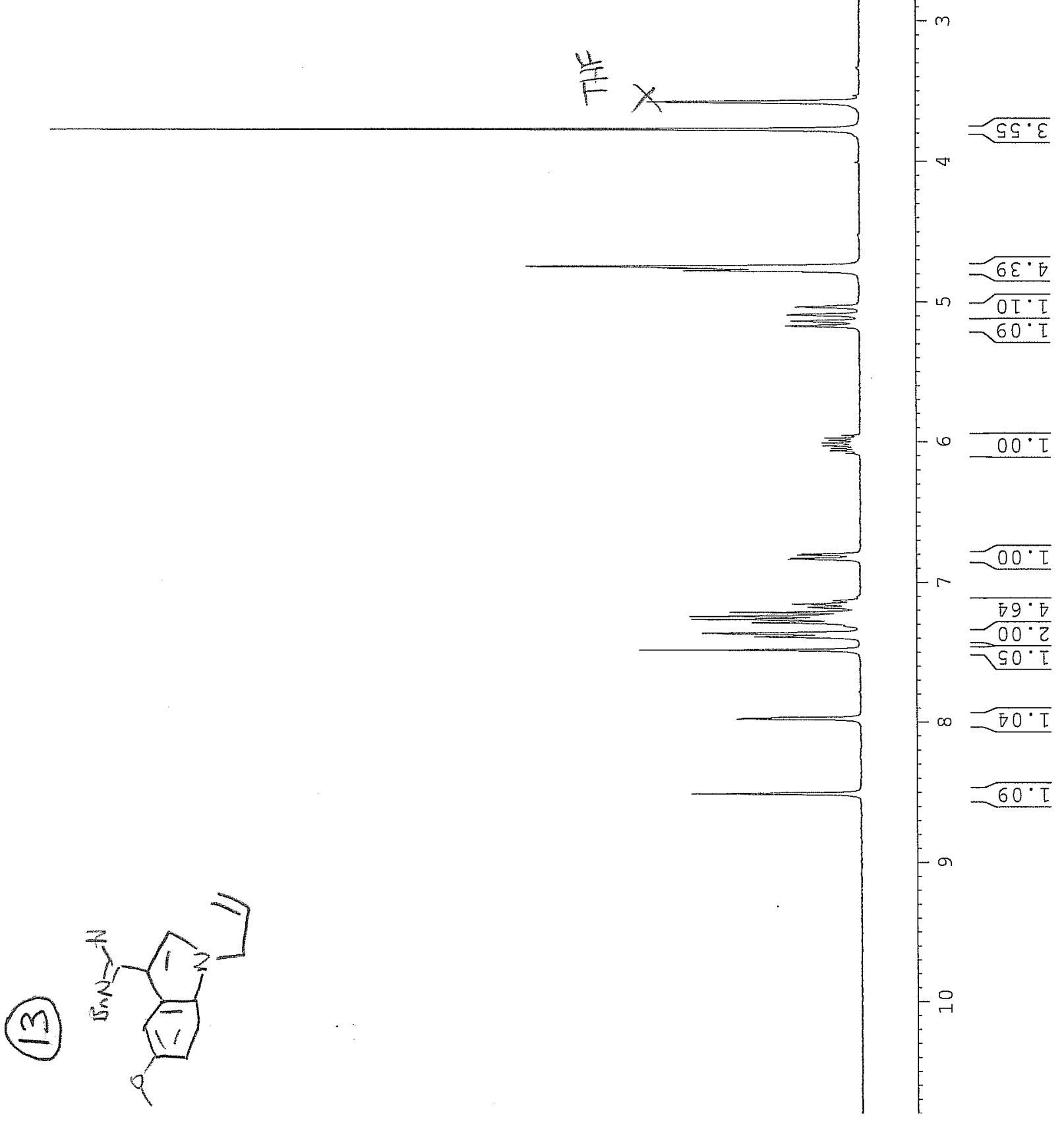



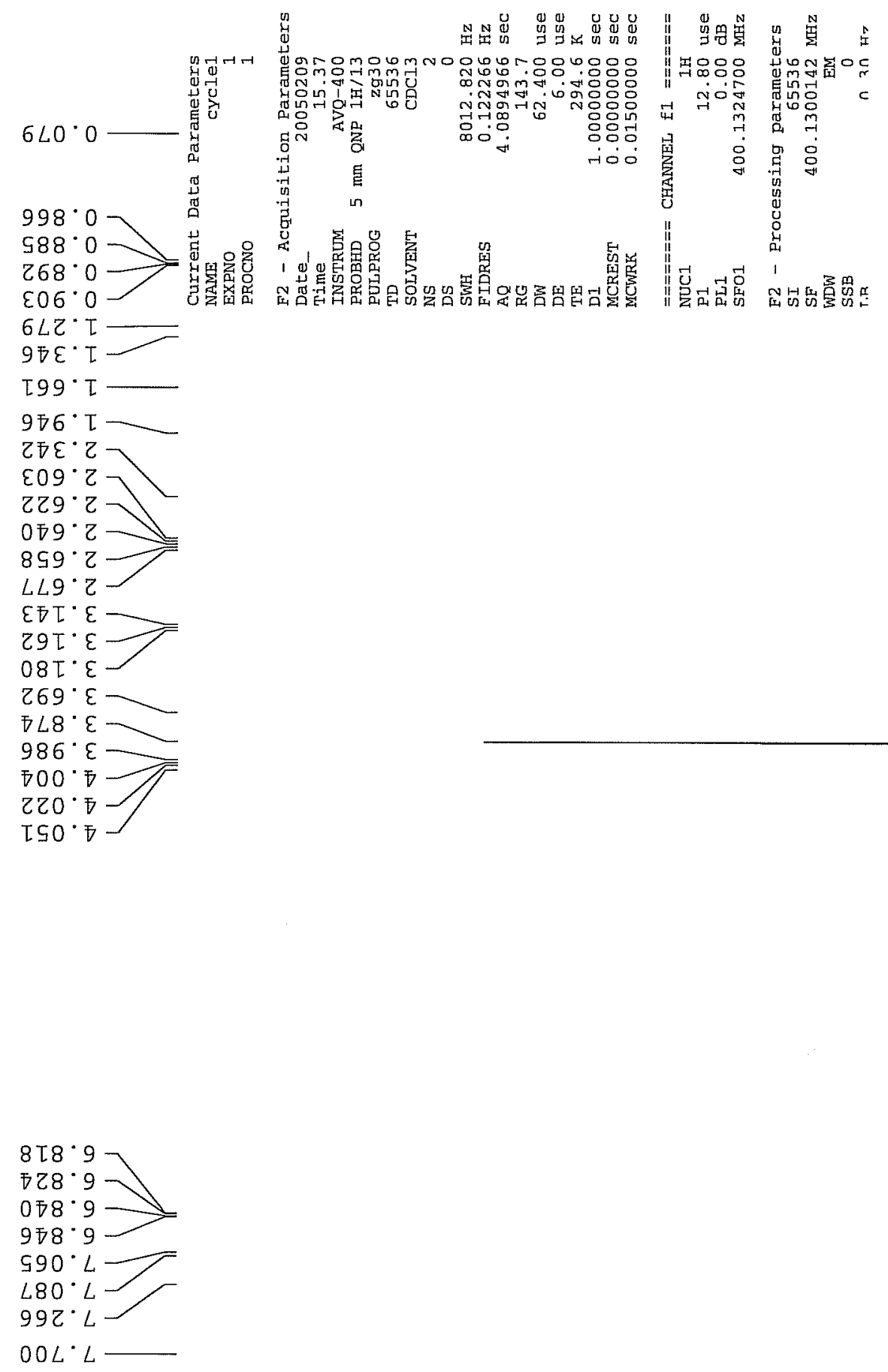

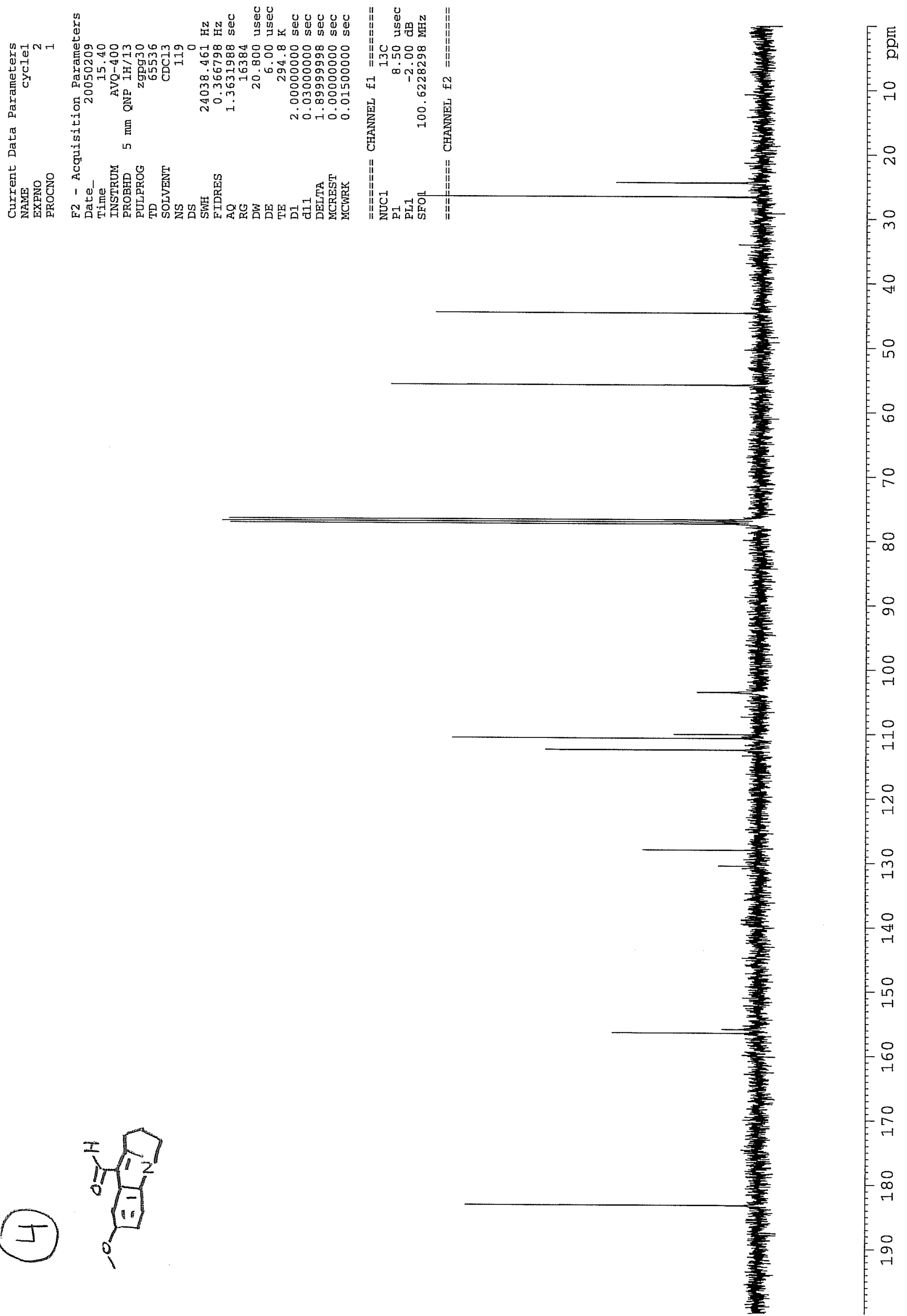


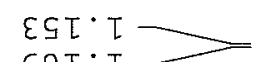

$69 \tau \cdot \tau \longrightarrow$

โ80.2

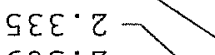
$605^{\circ} \mathrm{Z}$ LOL.
$\varepsilon Z L$
$L \varepsilon L$ $L E L \cdot Z \longrightarrow$ $L S L \cdot Z-$ $788^{\circ} \mathrm{r}$

$206^{\circ} \mathrm{Z}$

โ26.2 ५ $9 \varepsilon \cdot \varepsilon$

โ9L. $\varepsilon=$

$\varepsilon 86^{\circ} \varepsilon-$

$666^{\circ} \varepsilon \longrightarrow$

9โ0.

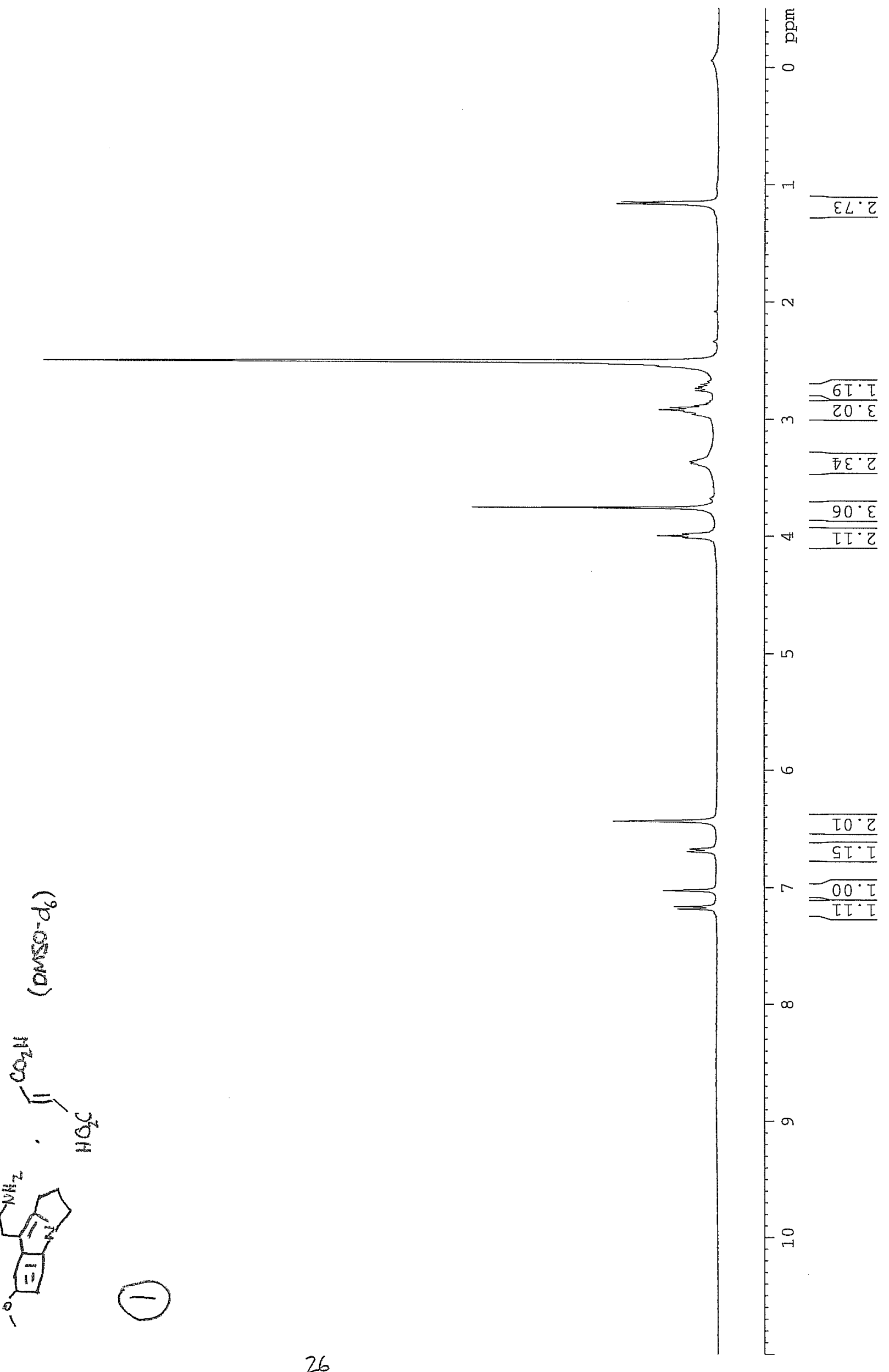

8Eี.9-

$2 \angle 9 \cdot 9 \longrightarrow$

$769 \cdot 9$

$\angle 20^{\circ} \mathrm{L}$

$79 I \cdot L=$

$98 \mathrm{~T} \cdot \mathrm{L} \longrightarrow$
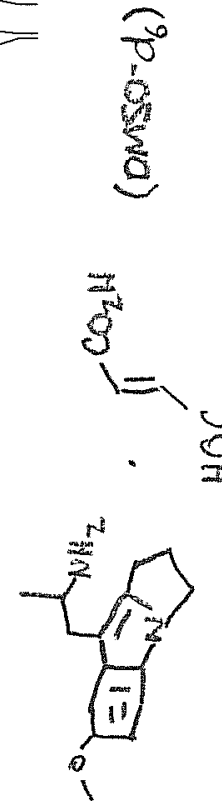


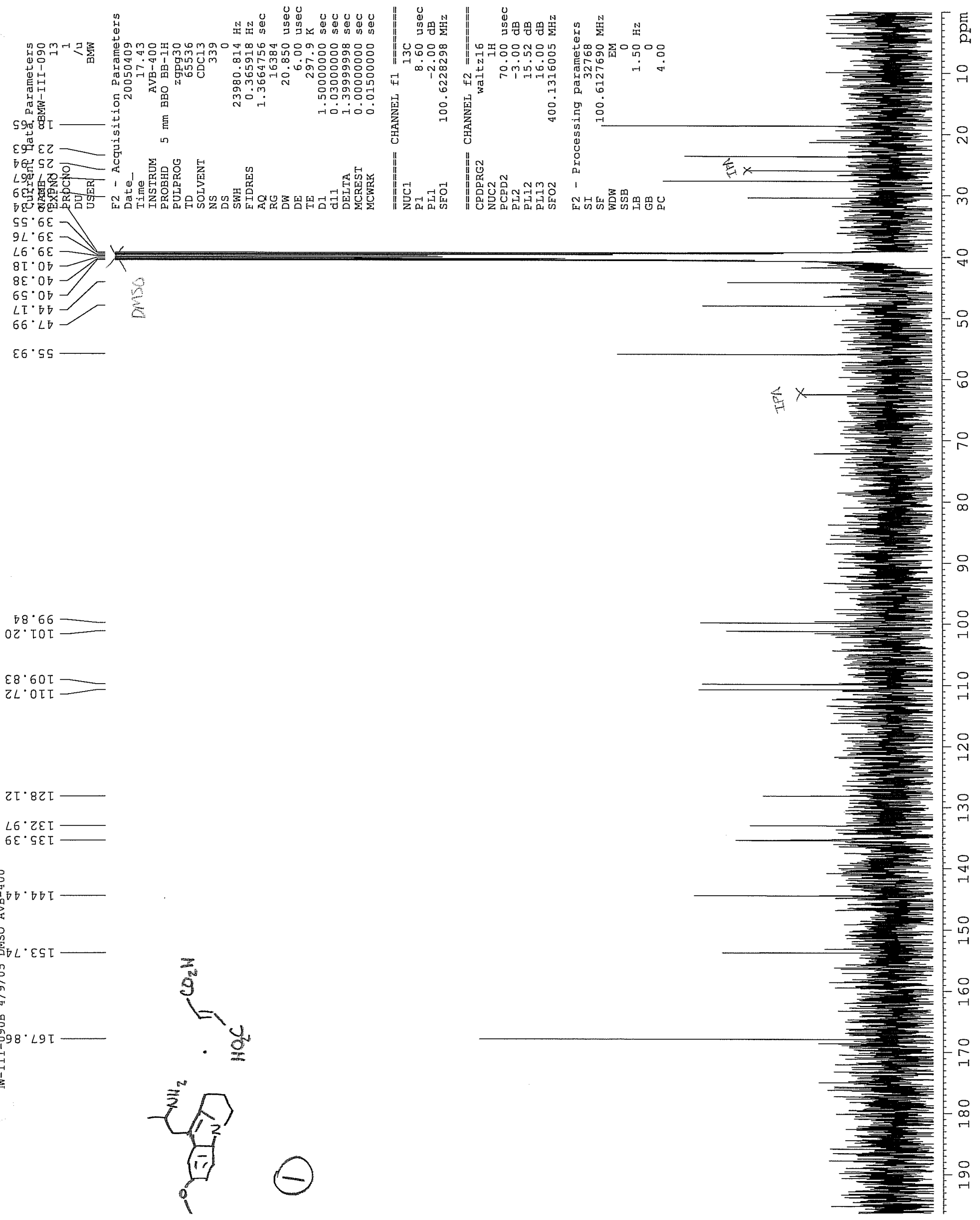




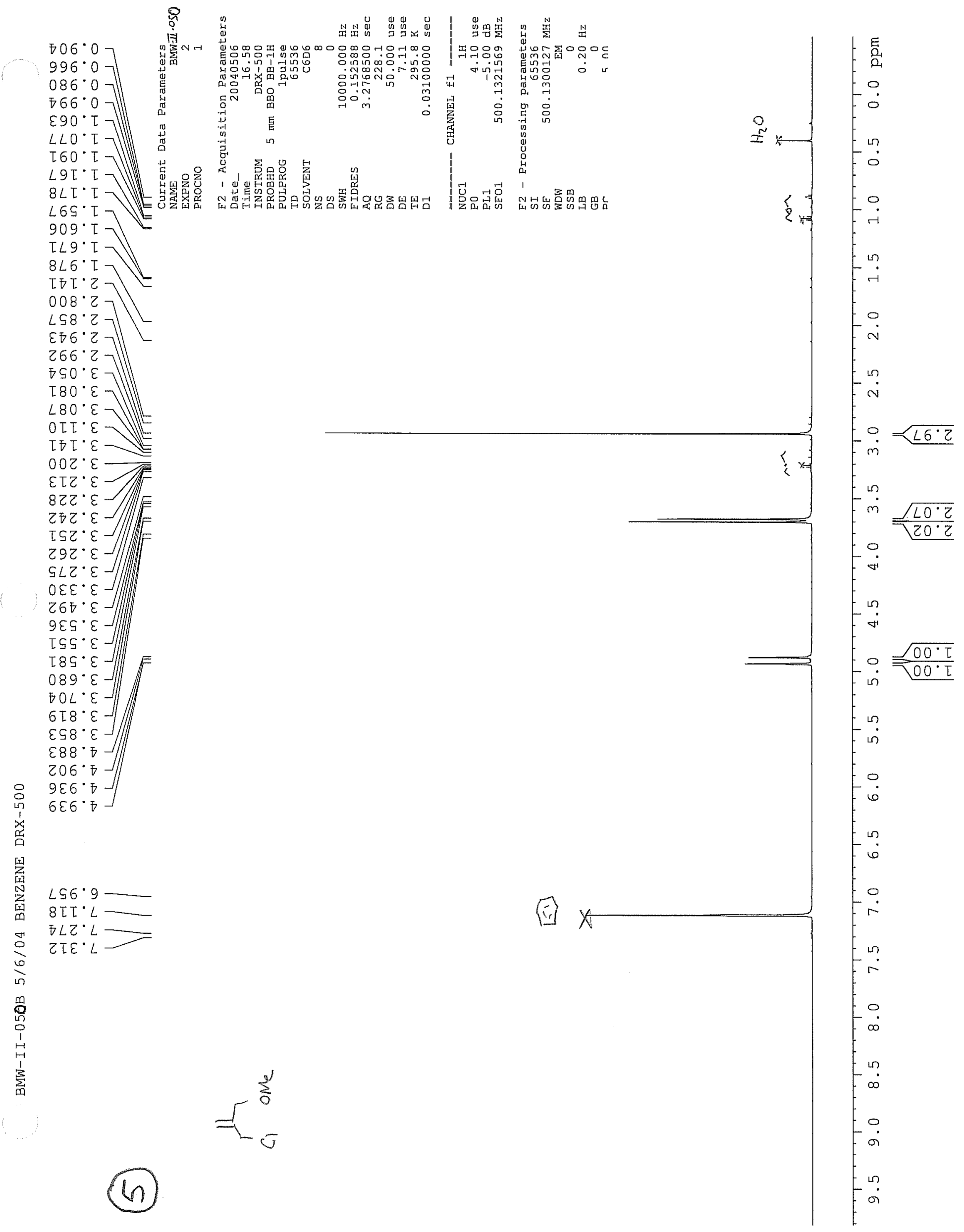



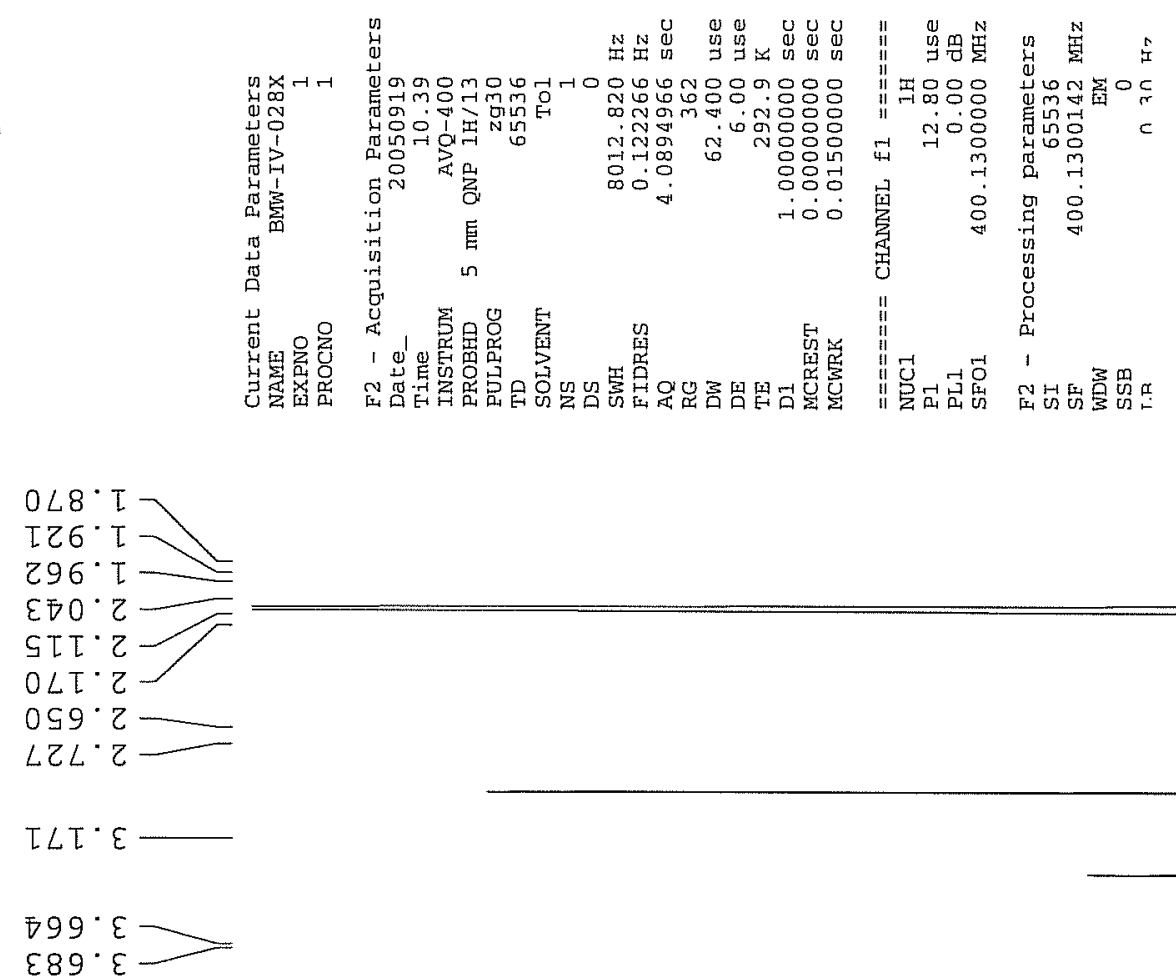

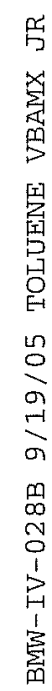

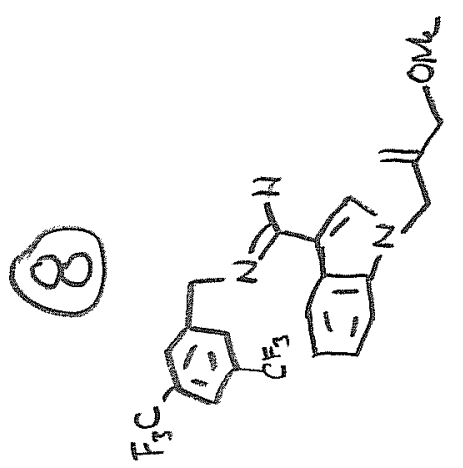




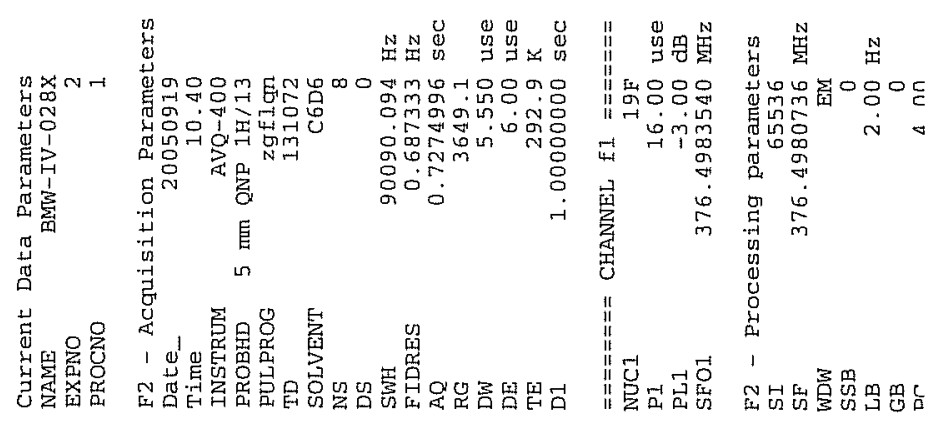

(0)

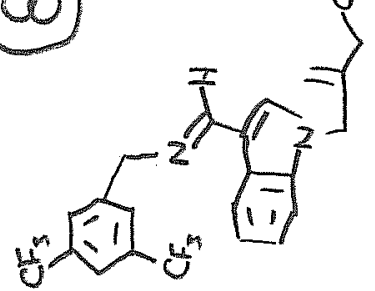



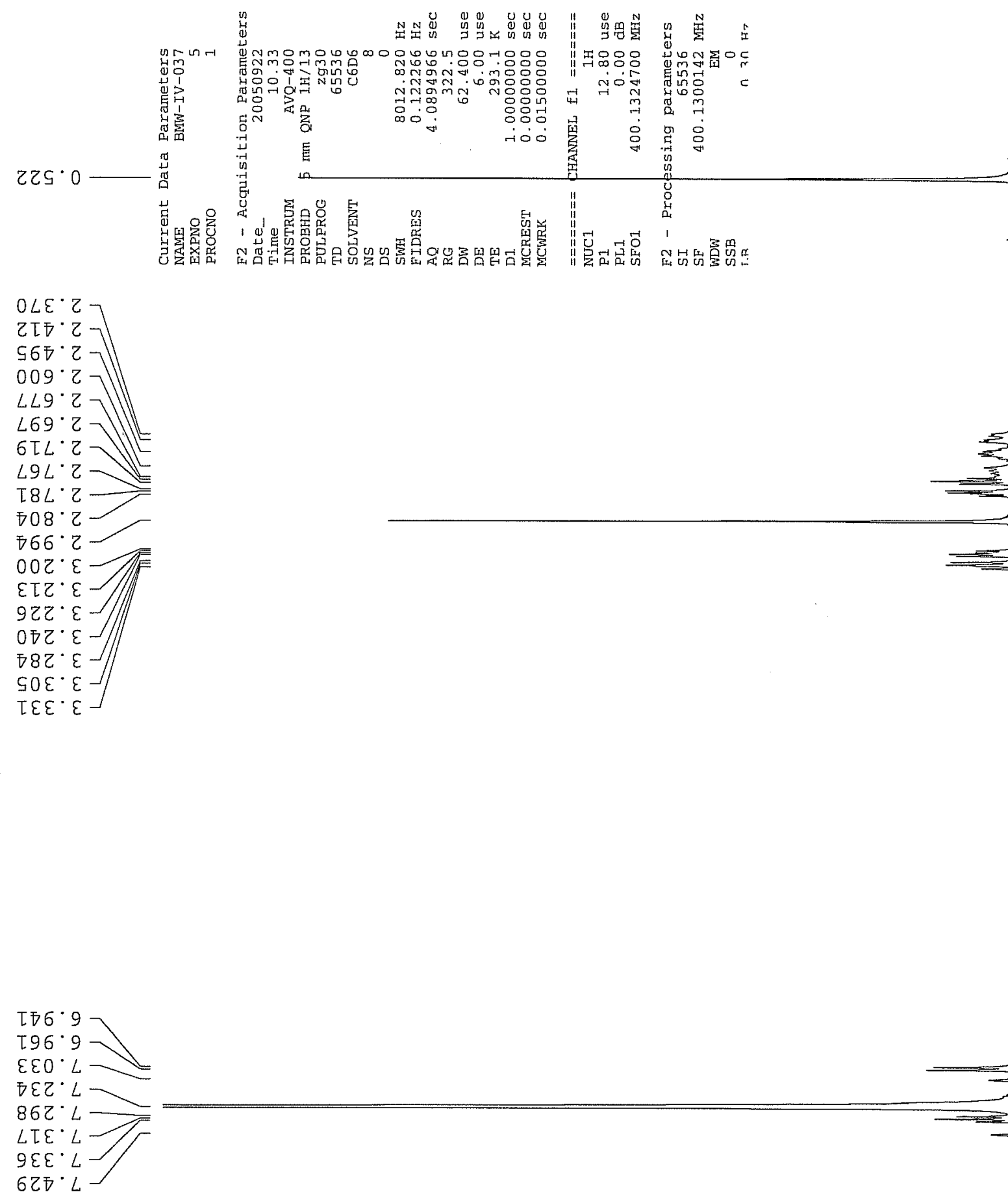

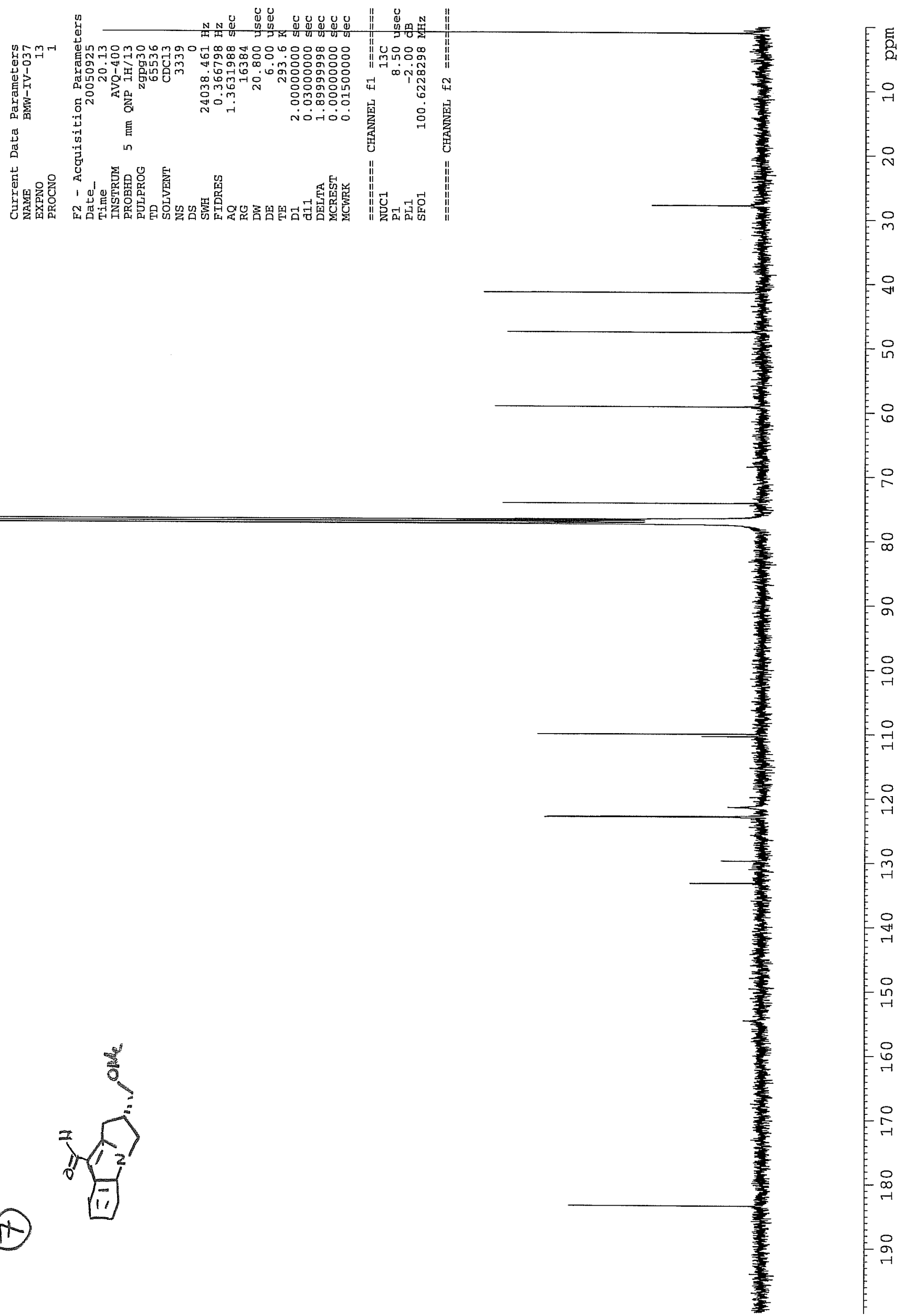


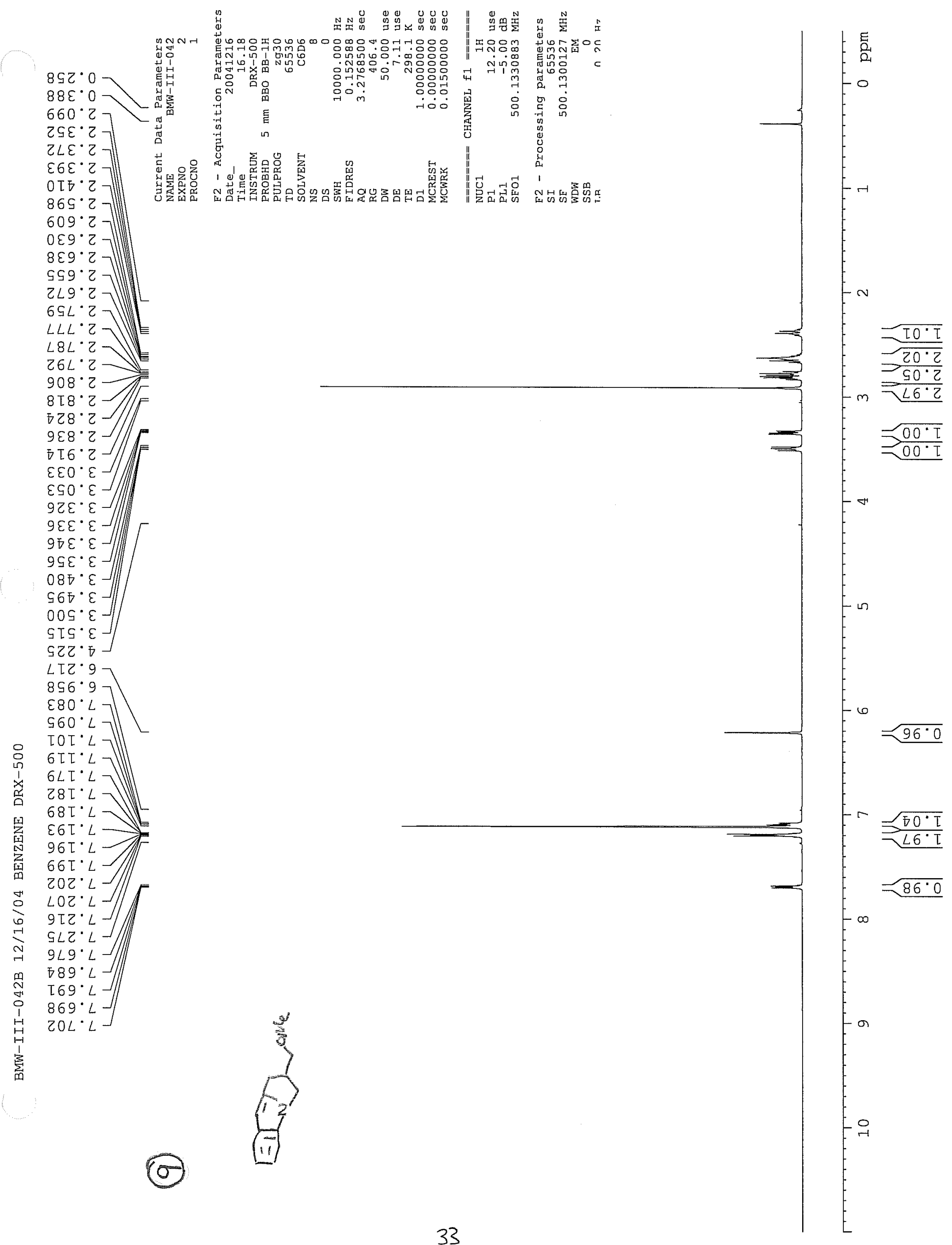




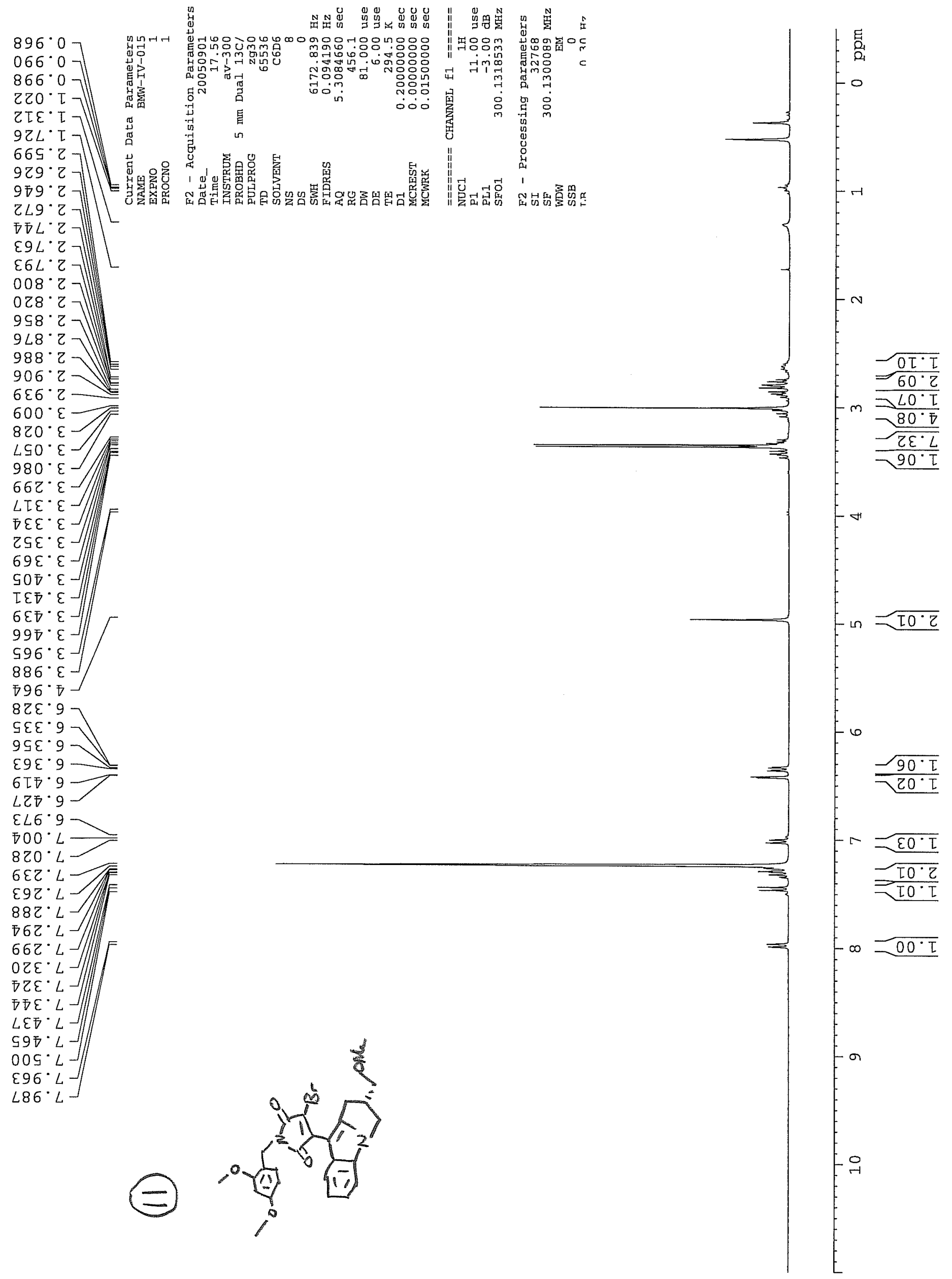




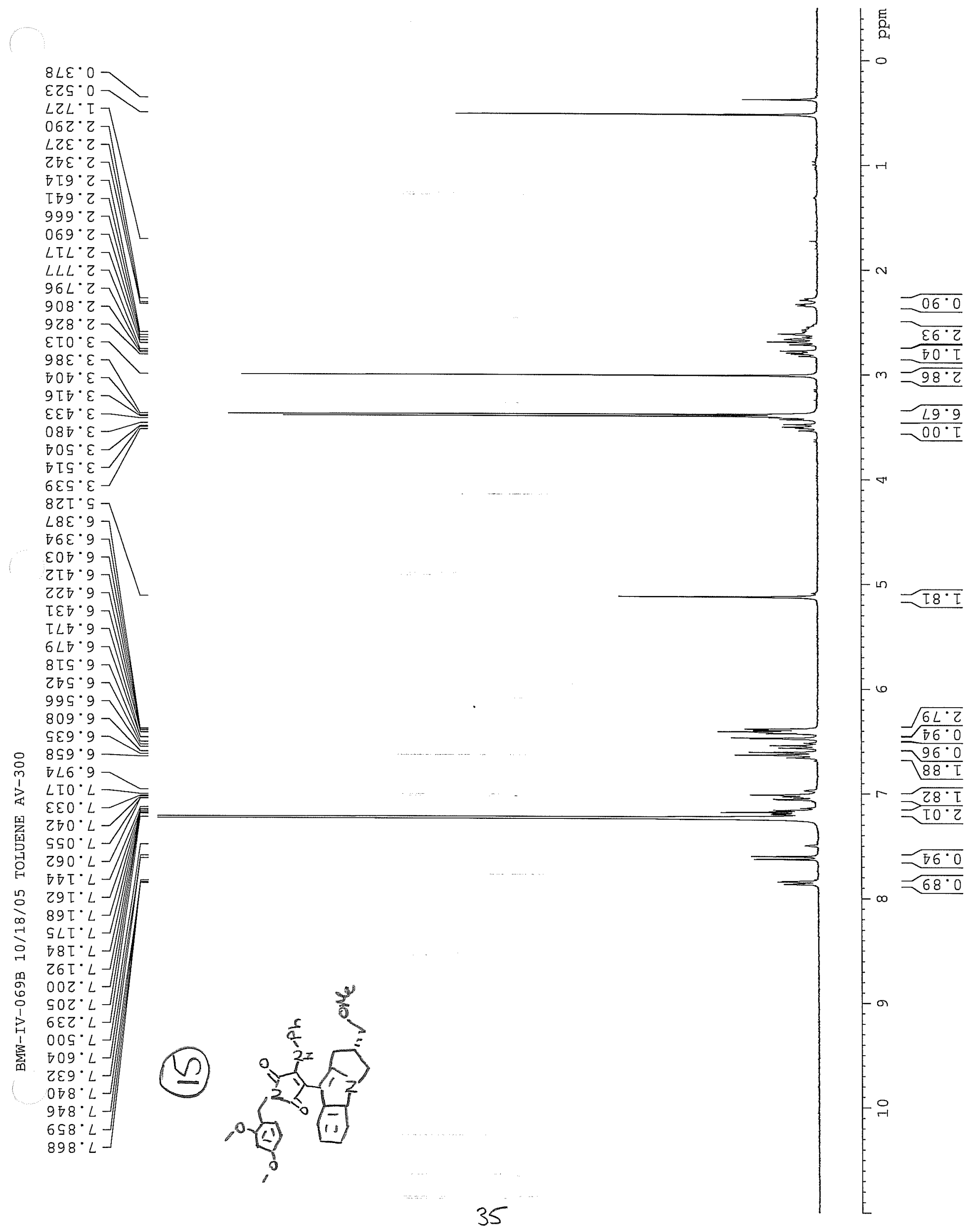




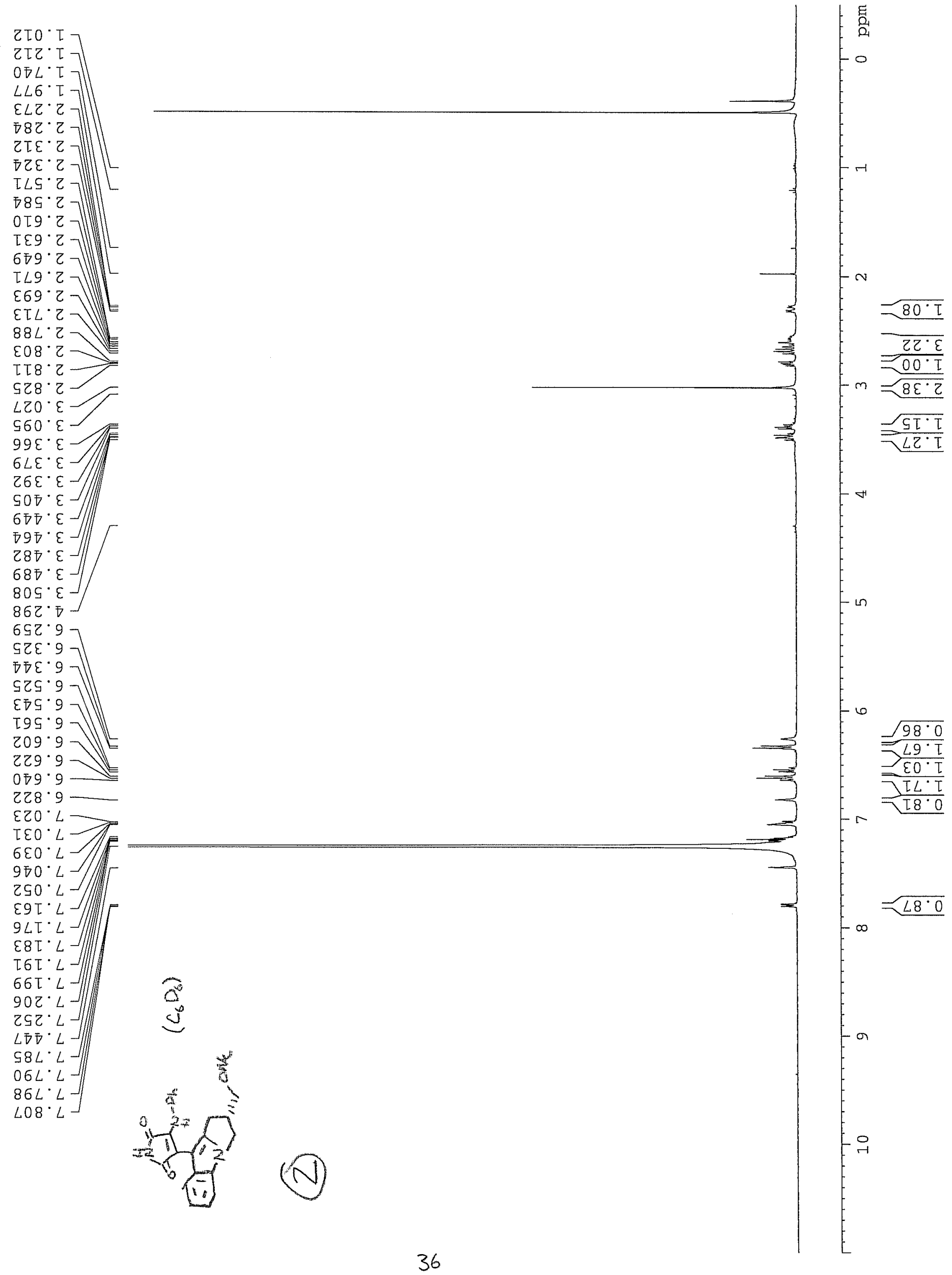




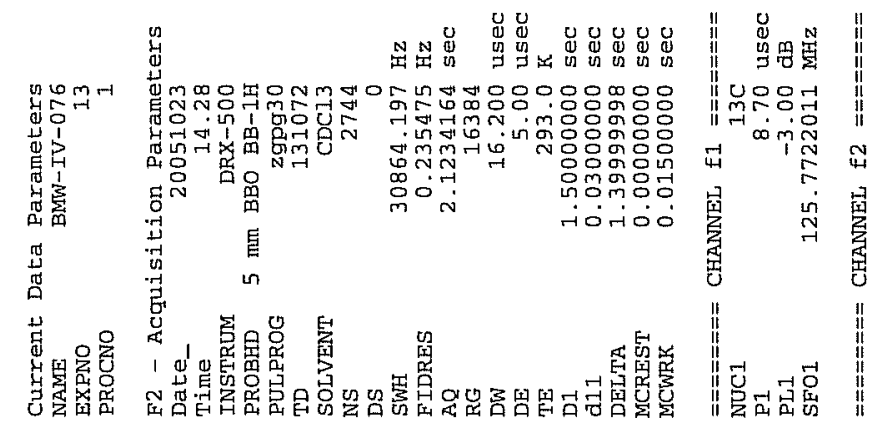

$78 \cdot 85$

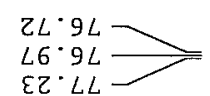

ZO.0ZT
$05^{\circ} \varepsilon Z T-$

$08 \cdot \angle 2 T$

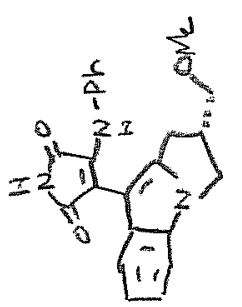

